\title{
Three-dimensional modeling and time-delay stability analysis for robotics docking simulation
}

\section{Prateek Sazawal ${ }^{1}$, Daniel Choukroun ${ }^{2}$, Heike Benninghoff ${ }^{3}$ and Eberhard Gill ${ }^{4}$}

\begin{abstract}
Hardware-in-the-loop (HIL) simulations of two interacting bodies are often accompanied by a time delay. The time delay, however small, may lead to instability in the HIL system. The present work investigates the source of instability in a two spacecraft system model with a time delayed contact force feedback. A generic compliance-device-based contact force model is proposed with elastic, viscous and Coulomb friction effects in three dimensions. A 3D nonlinear system model with time delay is simulated and the effect of variations in contact force model parameters is studied. The system is then linearized about a nominal state to determine the stability regions in terms of parameters of the spring-dashpot contact force model by the pole placement method. Furthermore, the stability analysis is validated for the nonlinear system by energy observation for both the stable and unstable cases.
\end{abstract}

\section{Keywords}

Contact model, Time-delay system, Compliance device, Hardware-in-the-loop docking simulator

\section{Introduction}

Rendezvous and Docking (RvD) is a mission-critical phase of current space missions to the International Space Station and of future missions for applications such as space debris removal and satellite-to-satellite refuelling ${ }^{1-3}$. RvD technologies are key to the development of OnOrbit Servicing (OOS) capabilities, which in turn will enable, among other objectives, satellite operational lifetime extension and performances improvement ${ }^{4-8}$. Various RvD testing set-ups are available for the ground emulation of space-like conditions ${ }^{9-13}$. The air-bearing test-beds are the most common for simulation and validation of systems with on-orbit applications. They have, however, physical limitations ${ }^{9,13}$. Robotics based RvD simulators, where multi-degrees of freedom robotic arms are employed in a Hardware-in-the-Loop (HIL) system, have obvious advantages, ${ }^{10,14,15}$ but also face known challenges such as time delays in the loop.

The interaction of two bodies in contact is central to the $\mathrm{RvD}$ simulations. Experiments have shown that for robotic impact control, a hardware compliance is a solution to low contact forces at the contact interface ${ }^{16}$. Mathematical models of contact dynamics serve as a tool for the analysis and evaluation of mechanical systems. Gilardi and Sharf (2002) ${ }^{17}$ presented a comprehensive review of the contact dynamic models in the literature. Earliest known is the Hertz contact model, a nonlinear model that is limited only to the elastic impacts without energy dissipation. The following models accounted for the energy dissipation by adding a velocity dependent damping term ${ }^{18,19}$. Bondoky et al. (2017) $)^{20}$ analyzed different contact dynamic models on an HIL testbed, and showed that the coefficient of restitution is the most influencing parameter for contact dynamics modeling. A linear spring-dashpot contact model is known for its relative simplicity and ease of analysis of dynamic systems in contact ${ }^{17}$. Spring-dashpot contact model, as well as an even simpler mass-spring model, has often been employed for analysis and evaluation of HIL simulations ${ }^{10,16,21-25}$.

\footnotetext{
${ }^{1}$ German Aerospace Center, German Space Operations Center, 82234 Wessling, Germany; Delft University of Technology, Faculty of Aerospace Engineering, 2629 HS, Delft, The Netherlands

${ }^{2}$ Ben-Gurion University of the Negev, Department of Mechanical Engineering, 84105, Beer Sheva, Israel (Email: danielch@bgu.ac.il) ${ }^{3}$ German Aerospace Center, German Space Operations Center, 82234 Wessling, Germany

${ }^{4}$ Delft University of Technology, Faculty of Aerospace Engineering, 2629 HS, Delft, The Netherlands
}

\section{Corresponding author:}

Prateek Sazawal, German Aerospace Center, German Space Operations Center, 82234 Wessling, Germany.

Email: prateeksazawal@gmail.com 
Combining time delays and high contact stiffness in a closed loop HIL simulator might cause instability, hardware damages, and eventually safety hazards ${ }^{26}$. Osaki et al. ${ }^{22}$ has shown analytically that a closed loop HIL system simulating an elastic collision does not obey energy conservation. To overcome the problem, time delay compensation for one dimensional HIL simulations has been addressed in several ways in the literature $20,22-25$. The present work, however, addresses this problem by determining a stability regions of the compliance parameters for the contact of two bodies moving in three dimensions.

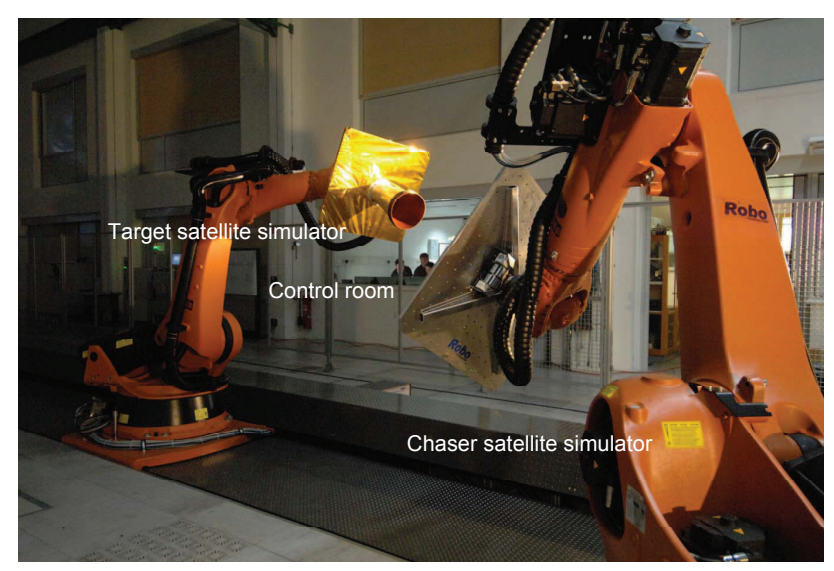

Figure 1. European Proximity Operations Simulator (EPOS) at GSOC, DLR ${ }^{10}$

The European Proximity Operations Simulator (EPOS) facility at the German Aerospace Center (DLR) is a robotics based HIL simulator, which allows testing and carrying out on-ground RvD simulation of two spacecraft ${ }^{15}$ as shown in Fig. (1). The verification of the docking phase of an OOS mission, particularly via a nozzle, is one of the aims of performing an RvD simulation on EPOS facility. It consists of two 6DOF industrial robots, one being stationary and the other one mounted on rails, giving a total of 13DOF. The robots at EPOS are capable of carrying payload masses up to $240 \mathrm{~kg}$ with a sub-millimeter and sub-degree position and pointing accuracy, respectively. The robot commands are issued to track the trajectory of simulated satellites in real time. The monitoring system of the facility is capable of measuring the current position and attitude of the robots. Efforts were conducted in developing a methodology for safe testing design at the $\operatorname{EPOS}^{10}$. Testing was performed and successfully validated the proposed stability analysis. An average time delay of $32 \mathrm{~ms}$ was observed due to communication channels during testing. The proposed work, however, was limited to a two-dimensional contact geometry, the testing was performed in a single-dimensional setting, and the physical scenario assumed a target satellite at rest with respect to the inertial frame.
This work builds on previous efforts while relaxing several assumptions. The contributions of this paper are as follows: 1) the Target-Chaser nonlinear Truth model is extended to three dimensions and features two moving frames, which is consistent with scenarios involving satellites of similar masses; 2) the contact dynamics model is extended and features a generic compliance device with three dimensional elastic, viscous, and Coulomb friction nonlinear effects; 3 ) the linear stability analysis is extended to a three dimensional space.

A realistic nonlinear Truth model is developed from first principles and brought to a reduced order state-space modeling of the satellites' and contact point motions. Simplifying physical assumptions leads to an intuitive model order reduction. The development of a linearized design model and physically motivated state transformation lay the ground for linear stability analysis of the penetration depth as well as other modes of the contact point motion. The stability analysis is performed via the pole placement method for linear time-delay systems. One convenient and elegant outcome of the proposed methodology is the similarity of the resulting 3D stability analysis with the work presented by Zebenay et al. (2015) ${ }^{10}$, thanks to the decoupled contact dynamics modes. All primal parameters, e.g. satellites' masses, inertias, nozzle geometry angle, are lumped within a smaller set of parameters. A numerical example illustrates the stability analysis results. Further, nonlinear simulations of the Truth model are performed in order to verify and validate the stability analysis. The trajectory of the Chaser's probe tip inside the Target's nozzle in three dimensions is simulated under various contact interface models and contact parameters values. An energy based method employed on the nonlinear model confirms the stability analysis results for the system under study.

The nonlinear system modeling along with the compliance device is discussed in the following section. The nonlinear system model is linearized with further reduction of system states. The stability analysis of the linearized model is carried out, and numerically validated for the nonlinear model.

\section{Truth Model}

A system of a Target and Chaser spacecraft is shown in Fig. (2). The Target spacecraft is mounted with a conical nozzle. The nozzle allows the probe of the Chaser spacecraft to route in and dock into the target. Points $T$ and $C$ are the center of masses of the Target and Chaser spacecraft, respectively. The inertial reference frame fixed to the laboratory, $\mathcal{G}$ has the origin at point $G$. The Target and Chaser spacecraft reference 
frames, $\mathcal{T}$ and $\mathcal{C}$, are respectively fixed to the Target and Chaser center of masses.

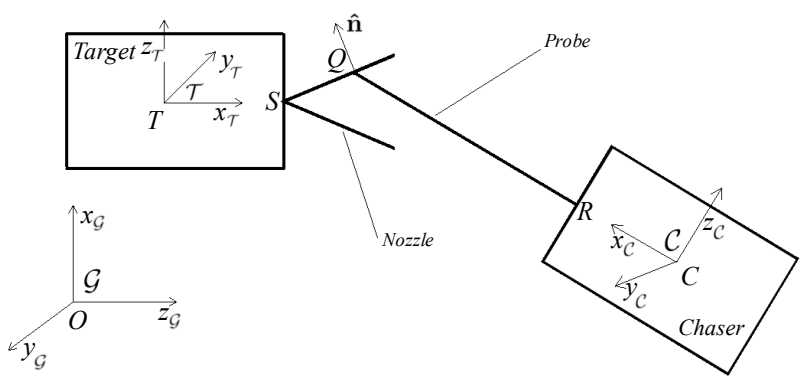

Figure 2. Target and Chaser spacecraft system

The direction cosine matrix is chosen as the attitude parameter, as it has an advantage of being a non-singular attitude representation of a body. $\mathbf{D}_{\mathcal{C}}^{\mathcal{G}}$ and $\mathbf{D}_{\mathcal{T}}^{\mathcal{G}}$ denote the direction cosine matrices from the inertial reference frame, $\mathcal{G}$, to the Chaser and Target reference frames, respectively. The positions, translational velocities, and angular velocities of the Chaser and Target with respect to $\mathcal{G}$ are denoted by $\mathbf{r}_{C}, \mathbf{r}_{T}, \mathbf{v}_{C}, \mathbf{v}_{T}, \omega_{C}$ and $\boldsymbol{\omega}_{T}$, respectively. The kinematic equations of motion are therefore written as follows

$$
\begin{aligned}
\dot{\mathbf{r}}_{C} & =\mathbf{v}_{C} \\
\dot{\mathbf{r}}_{T} & =\mathbf{v}_{T} \\
\dot{\mathbf{D}}_{\mathcal{C}}^{\mathcal{G}} & =-\omega_{C}^{\times} \mathbf{D}_{\mathcal{C}}^{\mathcal{G}} \\
\dot{\mathbf{D}}_{\mathcal{T}}^{\mathcal{G}} & =-\boldsymbol{\omega}_{T}^{\times} \mathbf{D}_{\mathcal{T}}^{\mathcal{G}}
\end{aligned}
$$

Assumption-1: The Chaser and Target spacecraft are modeled as rigid bodies. The dominant force and torque during contact between the Chaser and Target are the contact force and torque, and other causes are neglected. The contact force is applied at the tip of the probe, $Q$, which is modeled as a point.

Assumption-2: The deviation of the probe tip position from equilibrium relative to the Chaser Body is small.

The force and moment equations of the Chaser and Target spacecraft at the time of contact are given as follows

$$
\begin{aligned}
& m_{C} \dot{\mathbf{v}}_{C}=\mathbf{f} \\
& m_{T} \dot{\mathbf{v}}_{T}=-\mathbf{f} \\
& \mathbf{J}_{C} \dot{\boldsymbol{\omega}}_{C}=\mathbf{r}_{C Q}^{\times} \mathbf{D}_{\mathcal{C}}^{\mathcal{G}} \mathbf{f}-\boldsymbol{\omega}_{C}^{\times} \mathbf{J}_{C} \boldsymbol{\omega}_{C} \\
& \mathbf{J}_{T} \dot{\boldsymbol{\omega}}_{T}=-\mathbf{r}_{T Q}^{\times} \mathbf{D}_{\mathcal{T}}^{\mathcal{G}} \mathbf{f}-\boldsymbol{\omega}_{T}^{\times} \mathbf{J}_{T} \boldsymbol{\omega}_{T}
\end{aligned}
$$

where $m$ is the mass, $\mathbf{J}$ is the moment of inertia matrix, with subscripts $C$ and $T$ referring to the Chaser and Target, respectively. $\mathbf{f}$ is the contact force between the Chaser and Target expressed in inertial frame coordinates. $\mathbf{r}_{C Q}$ and $\mathbf{r}_{T Q}$ are the vectors $C Q$ and $T Q$ written in the Chaser and Target frame coordinates, respectively. The relationship between vectors $\mathbf{r}_{S Q}$ and $\mathbf{r}_{T Q}$ can be expressed in terms of the states of the system

$$
\mathbf{r}_{T Q}=\mathbf{D}_{\mathcal{T}}^{\mathcal{G}}\left[\mathbf{r}_{C}-\mathbf{r}_{T}+\mathbf{D}_{\mathcal{G}}^{\mathcal{C}} \mathbf{r}_{C Q}\right]
$$

It is to be noted that the force equations in (5) and (6) are written in the inertial reference frame coordinates, while the moment equations in (7) and (8) are written in Chaser and Target reference frame coordinates, respectively. The superscript $\times$ denotes the cross product skew symmetric matrix operator on the vector, such that

$$
\boldsymbol{\omega}^{\times}=\left[\begin{array}{ccc}
0 & -\omega_{3} & \omega_{2} \\
\omega_{3} & 0 & -\omega_{1} \\
-\omega_{2} & \omega_{1} & 0
\end{array}\right]
$$

Eqs. (1)-(8) constitute the equations of motion of the Chaser and Target spacecraft in a 36-dimensional state-space representation.

\section{Compliance device}

A compliance device is a hardware, providing a passive compliance to the impact due to contact between Chaser and Target bodies. In this section, equivalent dynamics are formulated for a generic compliance device model which contributes to the normal force at the contact point $Q$. The equivalent dynamics under specified assumptions are shown to be dependent on the system state. As the point of contact $Q$ is moving along the nozzle surface, another frame of reference, $\mathcal{N}$ is defined as shown in Fig. (3). The nozzle frame, $\mathcal{N}$ is defined keeping the origin fixed at point $S$, with the $x_{\mathcal{N}}$-axis along the vector $\mathbf{r}_{S Q}$ and the $z_{\mathcal{N}}$-axis along the local normal to the surface of the nozzle, $\hat{\mathbf{n}}$. The orientation of the nozzle frame with respect to the target frame is such that $x_{\mathcal{N}}$ makes an angle $\theta$ with $x_{\mathcal{T}}$ and $y_{\mathcal{N}}$ makes an angle $\phi$ with $y_{\mathcal{T}}$, therefore

$$
\mathbf{D}_{\mathcal{T}}^{\mathcal{N}}=\left[\begin{array}{ccc}
\cos \theta & 0 & -\sin \theta \\
-\sin \theta \sin \phi & \cos \phi & -\cos \theta \sin \phi \\
\sin \theta \cos \phi & \sin \phi & \cos \theta \cos \phi
\end{array}\right]
$$

where $\phi \in[0,2 \pi)$ is the azimuthal angle and $\theta$ is the constant semi-cone angle of the nozzle. $\hat{\mathbf{m}}$ and $\hat{\mathbf{t}}$ denote the unit vectors in radial direction, along the $x_{\mathcal{N}}$ axis, and in tangential direction, along the $y_{\mathcal{N}}$ axis, respectively.

The contact force at the nozzle surface is a resultant of the reaction forces normal to the surface, $\mathbf{f}_{n}$, and along the surface, $\mathbf{f}_{n_{\perp}}$, as follows 


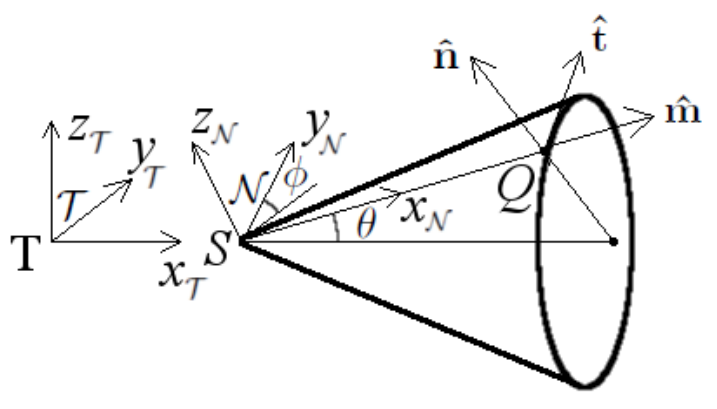

Figure 3. Nozzle reference frame

$$
\mathbf{f}=\mathbf{f}_{n}+\mathbf{f}_{n_{\perp}}
$$

Note that $\mathbf{f}, \mathbf{f}_{n}$, and $\mathbf{f}_{n_{\perp}}$ are written in inertial frame coordinates. If $\hat{\mathbf{n}}$ and $\hat{\mathbf{n}}_{\perp}$ respectively denote the unit vectors along the two mutually perpendicular reaction forces expressed in the Nozzle frame, $\mathcal{N}$

$$
\begin{aligned}
\mathbf{f}_{n} & =f_{n} \mathbf{D}_{\mathcal{G}}^{\mathcal{N}} \hat{\mathbf{n}} \\
\mathbf{f}_{n_{\perp}} & =f_{n_{\perp}} \mathbf{D}_{\mathcal{G}}^{\mathcal{N}} \hat{\mathbf{n}}_{\perp}
\end{aligned}
$$

The sliding of contact point $Q$ on the nozzle surface gives rise to a force of friction between the Chaser and Target bodies.

Assumption-3: The force of friction is along the surface of the nozzle, ${ }^{17,18,27}$ and modeled according to the Coulomb friction model.

The contact force along the nozzle surface can be written as

$$
f_{n_{\perp}}=\mu f_{n}
$$

where $\mu$ is the coefficient of kinetic friction between the nozzle surface and the probe tip. The direction of the friction force is opposite to the sliding velocity of point $Q$ over the nozzle surface, given as

$$
\hat{\mathbf{n}}_{\perp}=\mathbf{D}_{\mathcal{N}}^{\mathcal{T}} \frac{\left(\mathbf{I}-\mathbf{n}_{\mathcal{T}} \mathbf{n}_{\mathcal{T}}^{T}\right) \dot{\mathbf{r}}_{S Q}}{\left\|\left(\mathbf{I}-\mathbf{n}_{\mathcal{T}} \mathbf{n}_{\mathcal{T}}^{T}\right) \dot{\mathbf{r}}_{S Q}\right\|}
$$

where $\mathbf{I}$ is the identity matrix and $\mathbf{n}_{\mathcal{T}}=\mathbf{D}_{\mathcal{T}}^{\mathcal{N}} \hat{\mathbf{n}}$ is the unit vector normal to the nozzle surface at the point of contact, as expressed in the Target fixed frame. The time derivative of vector $\mathbf{r}_{S Q}$ results from Eq. (9)

$$
\dot{\mathbf{r}}_{S Q}=\dot{\mathbf{r}}_{T Q}=-\boldsymbol{\omega}_{T}^{\times} \mathbf{r}_{T Q}+\mathbf{D}_{\mathcal{T}}^{\mathcal{G}}\left[\mathbf{v}_{C}-\mathbf{v}_{T}+\mathbf{D}_{\mathcal{G}}^{\mathcal{C}} \boldsymbol{\omega}_{C}^{\times} \mathbf{r}_{C Q}\right]
$$

where $\mathbf{r}_{C Q}$ is assumed constant, following Assumption-2.

Figure (4) shows a compliance device mounted on the Chaser spacecraft. The compliance device model consists of an assembly of springs converging at point $P$ on the probe $R Q$. Note that the spring linkage between points $R$ and $P$ is

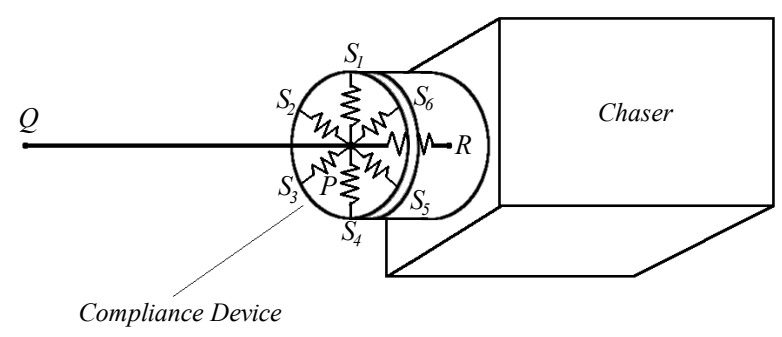

Figure 4. Chaser equipped with a compliance device

to provide a compliance if there is an impact along the probe length and normal to the nozzle surface.

Assumption-4: Each spring exerts a restoring force proportional to its relative longitudinal displacement.

Figure (5) shows the deviation of the probe $R P Q$ from the equilibrium position $R P_{o} Q_{o}$. A probe fixed frame $\mathcal{P}$ is defined as shown in Fig. (5) with $R$ as the origin, such that $x_{\mathcal{P}}$ is along the probe longitudinal axis. The axis $z_{\mathcal{P}}$ is perpendicular to $x_{\mathcal{P}}$ and lying in the plane containing $R, P$ and $P_{o}$, and $y_{\mathcal{P}}$ is the third axis of the right hand coordinate system. The probe frame $\mathcal{P}$ can be obtained by successively rotating the frame $\mathcal{C}$ by an angle $\phi_{\mathcal{P}}$ about the $x$ axis and by an angle $\theta_{\mathcal{P}}$ about the $y$ axis.

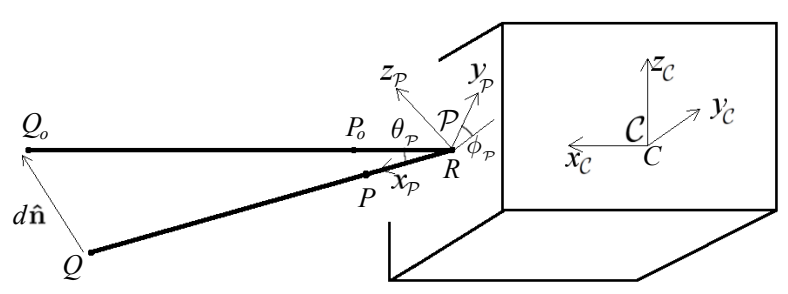

Figure 5. Compliance device model

If the displacement of point $Q$ is $d$ along the direction normal to the surface of the nozzle, then the following can be stated

$$
\mathbf{r}_{R Q}=l_{0} \hat{\mathbf{x}}_{\mathcal{C}}-d \mathbf{D}_{\mathcal{C}}^{\mathcal{N}} \hat{\mathbf{n}}
$$

where, $\mathbf{r}_{R Q}$ is the vector along $R Q$ written in the Chaser frame coordinates, $l_{0}$ is the length of the probe at equilibrium and $\hat{\mathbf{x}}_{\mathcal{C}}=\{1,0,0\}^{T}$ is the unit vector along the $\mathrm{x}$ axis of the Chaser. The length of the probe and the unit vector along the probe at any time is given by

$$
\begin{aligned}
& l=\left\|\mathbf{r}_{R Q}\right\| \\
& \hat{\mathbf{l}}=\frac{\mathbf{r}_{R Q}}{l}
\end{aligned}
$$

where $\hat{l}$ is the unit vector along the probe length written in the Chaser coordinate frame. Let $a$ denotes the ratio of the length 
of $P Q$ and the length of the probe at equilibrium, therefore

$$
\begin{aligned}
\mathbf{r}_{R P} & =\mathbf{r}_{R Q}-\mathbf{r}_{P Q} \\
& =l_{0} \hat{\mathbf{x}}_{\mathcal{C}}-d \mathbf{D}_{\mathcal{C}}^{\mathcal{N}} \hat{\mathbf{n}}-a l_{0} \hat{\mathbf{l}}
\end{aligned}
$$

Using Eq. (20), the shift in the position of point $P$ with respect to the Chaser, that is vector $P_{o} P$, can be written as

$$
\delta \mathbf{r}_{R P}=-d \hat{\mathbf{n}}_{\mathcal{C}}-a l_{0}\left[\hat{\mathbf{1}}-\hat{\mathbf{x}}_{\mathcal{C}}\right]
$$

where $\hat{\mathbf{n}}_{\mathcal{C}}=\mathbf{D}_{\mathcal{C}}^{\mathcal{N}} \hat{\mathbf{n}}$. Therefore, the elongation of the spring$i, \delta s_{i}$ can be expressed as the projection of $\delta \mathbf{r}_{R P}$ on the unit vector along the spring

$$
\delta s_{i}=\hat{\mathbf{s}}_{i}^{T} \delta \mathbf{r}_{R P}
$$

where $\hat{\mathbf{s}}_{i}$ is the inward unit vector along the spring written in the Chaser frame coordinates

$$
\hat{\mathbf{s}}_{i}=\frac{\mathbf{r}_{R P}-\mathbf{r}_{R S_{i}}}{\left\|\mathbf{r}_{R P}-\mathbf{r}_{R S_{i}}\right\|}
$$

and $\mathbf{r}_{R S_{i}}$ are the constant vectors $R S_{i}$ as shown in Fig. (4). If $k_{i}$ is the stiffness of the $i^{t h}$ spring, the force experienced by the probe due to spring $i$ is given by

$$
\mathbf{f}_{s i}=-k_{i} \delta s_{i} \hat{\mathbf{s}}_{i}
$$

Substituting Eqs. (21) and (22) in Eq. (24) and summing over $N$ springs

$$
\mathbf{f}_{s}=-\sum_{i=1}^{N} k_{i}\left(\hat{\mathbf{s}}_{i} \hat{\mathbf{s}}_{i}^{T}\right)\left[-d \hat{\mathbf{n}}_{\mathcal{C}}-a l_{0} \hat{\mathbf{l}}+a l_{0} \hat{\mathbf{x}}_{\mathcal{C}}\right]
$$

Equation (25) gives the net spring force $\mathbf{f}_{s}$ acting on the probe due to the Chaser body. As the probe is continuously under contact with the Target body at point $Q$, the projection of the net spring force on $\hat{\mathbf{n}}$ leads to the normal force $\mathbf{f}_{n}$

$$
\begin{aligned}
f_{n} & =-\hat{\mathbf{n}}_{\mathcal{C}}^{T} \mathbf{f}_{s} \\
& =\sum_{i=1}^{N} k_{i}\left(\hat{\mathbf{n}}_{\mathcal{C}}^{T} \hat{\mathbf{s}}_{i}\right) \hat{\mathbf{s}}_{i}^{T}\left[-d \hat{\mathbf{n}}_{\mathcal{C}}-a l_{0} \hat{\mathbf{l}}+a l_{0} \hat{\mathbf{x}}_{\mathcal{C}}\right]
\end{aligned}
$$

Assumption-5: The generic compliance device model only contributes to the force normal to the nozzle surface.

Under Assumption-5, the projection of the net spring force on $\hat{\mathbf{n}}$ is the only force relevant to the present model, and therefore the component along the nozzle surface is discarded. Substituting î from Eq. (19) leads to

$$
\begin{aligned}
f_{n}= & -\left(1-a \frac{l_{0}}{l}\right)\left[\sum_{i=1}^{N} k_{i}\left(\hat{\mathbf{n}}_{\mathcal{C}}^{T} \hat{\mathbf{s}}_{i}\right)^{2}\right] d \\
& -a l_{0}\left(\frac{l_{0}}{l}-1\right)\left[\sum_{i=1}^{N} k_{i}\left(\hat{\mathbf{n}}_{\mathcal{C}}^{T} \hat{\mathbf{s}}_{i}\right)\left(\hat{\mathbf{x}}_{\mathcal{C}}^{T} \hat{\mathbf{s}}_{i}\right)\right]
\end{aligned}
$$

\section{Contact point motion}

The location of point $Q$ on the nozzle can be solely determined from the position and attitude of Chaser and Target. A set of three parameters

$$
(d, m, \phi)
$$

defines the position point $Q$ on the nozzle surface, where $m$ is the distance of point $Q$ from the origin of Nozzle frame and $\phi$ is the azimuthal angle of Nozzle frame. The displacement of $Q$ along local normal to the nozzle surface, $d$, is attributed to the penetration in the nozzle surface, and is termed as penetration depth in the rest of the article.

If $\mathbf{r}_{S Q}$ denotes the vector $S Q$ expressed in Target frame coordinates, it follows from above that

$$
\mathbf{r}_{S Q}=\mathbf{D}_{\mathcal{T}}^{\mathcal{N}}[m \hat{\mathbf{m}}+d \hat{\mathbf{n}}]
$$

where, $\hat{\mathbf{m}}$ is the unit vector along the radial axis $x_{\mathcal{N}}$. The azimuthal angle can be found by substituting Eq. (11) in (29) and taking the projection of $\mathbf{r}_{S Q}$ along $y$ and $z$ axes of the Target frame

$$
\tan \phi=-\frac{\hat{\mathbf{y}}_{\mathcal{T}}^{T} \mathbf{r}_{S Q}}{\hat{\mathbf{z}}_{\mathcal{T}}^{T} \mathbf{r}_{S Q}}, \quad \quad \hat{\mathbf{z}}_{\mathcal{T}}^{T} \mathbf{r}_{S Q} \neq 0
$$

where $\hat{\mathbf{y}}_{\mathcal{T}}=(0,1,0)^{T}$ and $\hat{\mathbf{z}}_{\mathcal{T}}=(0,0,1)^{T}$ are the unit vectors along $y_{\mathcal{T}}$ and $z_{\mathcal{T}}$ axes respectively, and from Eq. (9)

$$
\begin{aligned}
\mathbf{r}_{S Q} & =\mathbf{r}_{T Q}-\mathbf{r}_{T S} \\
& =\mathbf{D}_{\mathcal{T}}^{\mathcal{G}}\left[\mathbf{r}_{C}-\mathbf{r}_{T}+\mathbf{D}_{\mathcal{G}}^{\mathcal{C}} \mathbf{r}_{C Q}\right]-\mathbf{r}_{T S}
\end{aligned}
$$

As the probe penetrates the nozzle, the point $Q$ moves in a direction along the normal to the nozzle surface, such that

$$
d=\left[\mathbf{D}_{\mathcal{N}}^{\mathcal{T}} \mathbf{r}_{S Q}\right]^{T} \hat{\mathbf{n}}
$$

where $\mathbf{r}_{S Q}$ is the vector $S Q$ expressed in Target frame coordinates. Substituting $\mathbf{r}_{S Q}$ from Eqs. (31) yields

$$
d=\left[\mathbf{D}_{\mathcal{N}}^{\mathcal{G}}\left(\mathbf{r}_{C}-\mathbf{r}_{T}\right)+\mathbf{D}_{\mathcal{N}}^{\mathcal{C}} \mathbf{r}_{C Q}-\mathbf{D}_{\mathcal{N}}^{\mathcal{T}} \mathbf{r}_{T S}\right]^{T} \hat{\mathbf{n}}
$$

where $\mathbf{r}_{T S}$ is a constant vector $T S$ expressed in the Target frame coordinates. 
Let $\mathbf{r}$ and $\mathbf{v}$ denote the relative position and velocity of the Chaser with respect to the Target, respectively, that is

$$
\begin{gathered}
\mathbf{r}=\mathbf{r}_{C}-\mathbf{r}_{T} \\
\mathbf{v}=\mathbf{v}_{C}-\mathbf{v}_{T}
\end{gathered}
$$

Using Eq. (34) in Eqs. (1)-(9) and (33) yields

$$
\begin{gathered}
\dot{\mathbf{r}}=\mathbf{v} \\
\dot{\mathbf{v}}=\frac{\mathbf{f}}{m_{r}} \\
\dot{\mathbf{D}}_{\mathcal{C}}^{\mathcal{G}}=-\boldsymbol{\omega}_{C}^{\times} \mathbf{D}_{\mathcal{C}}^{\mathcal{G}} \\
\dot{\mathbf{D}}_{\mathcal{T}}^{\mathcal{G}}=-\boldsymbol{\omega}_{T}^{\times} \mathbf{D}_{\mathcal{T}}^{\mathcal{G}} \\
\dot{\boldsymbol{\omega}}_{C}=\mathbf{J}_{C}^{-1}\left[\mathbf{r}_{C Q}^{\times} \mathbf{D}_{\mathcal{C}}^{\mathcal{G}} \mathbf{f}-\boldsymbol{\omega}_{C}^{\times} \mathbf{J}_{C} \boldsymbol{\omega}_{C}\right] \\
\dot{\boldsymbol{\omega}}_{T}=\mathbf{J}_{T}^{-1}\left[-\mathbf{r}_{T Q}^{\times} \mathbf{D}_{\mathcal{T}}^{\mathcal{G}} \mathbf{f}-\boldsymbol{\omega}_{T}^{\times} \mathbf{J}_{T} \boldsymbol{\omega}_{T}\right] \\
\mathbf{r}_{T Q}=\mathbf{D}_{\mathcal{T}}^{\mathcal{G}}\left[\mathbf{r}+\mathbf{D}_{\mathcal{G}}^{\mathcal{C}} \mathbf{r}_{C Q}\right] \\
d=\mathbf{r}^{T} \mathbf{D}_{\mathcal{G}}^{\mathcal{N}} \hat{\mathbf{n}}+\mathbf{r}_{C Q}^{T} \mathbf{D}_{\mathcal{C}}^{\mathcal{N}} \hat{\mathbf{n}}-\mathbf{r}_{T S}^{T} \mathbf{D}_{\mathcal{T}}^{\mathcal{N}} \hat{\mathbf{n}}
\end{gathered}
$$

where $m_{r}=\frac{m_{C} m_{T}}{m_{C}+m_{T}}$ is the reduced mass of the system in Eq. (36). Since the resulting equations only feature the vectors $\mathbf{r}$ and $\mathbf{v}$, they are used to define a new set of state variables. This conveniently reduces the dimension of the system from 36 down to 30 . It should be emphasized that defining the relative position and velocity as the state variables does not limit the scope of the analysis of the system in the following sections.

\section{Time-delayed system}

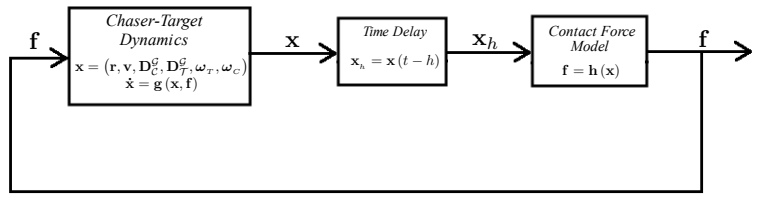

Figure 6. Block diagram of time delayed system model

The HIL Rendezvous and Docking Simulation is accompanied by a time delay due to communication channels as shown in Fig. (6). Hence, the position and attitude commands, $\mathbf{x}$, determined from the contact force and the Chaser-Target dynamics correspond to the state of the system at an earlier time. Assuming a time delay of $h$ seconds, the contact force given in Eq. (12) must be modified as

$$
\mathbf{f}(t)=\mathbf{f}_{n}(t-h)+\mathbf{f}_{n_{\perp}}(t-h)
$$

where $\mathbf{f}_{n}(t-h)$ and $\mathbf{f}_{n_{\perp}}(t-h)$ are the contact force components normal and along the nozzle surface, respectively.
The force feedback into the simulation at the current time is denoted by $\mathbf{f}(t)$.

\section{Truth model summary}

The time delayed system model in the state-space form can be summarized using Eqs. (1)-(8) as follows

$$
\begin{gathered}
\dot{\mathbf{r}}=\mathbf{v} \\
\dot{\mathbf{v}}=\frac{\mathbf{f}}{m_{r}} \\
\dot{\mathbf{D}}_{\mathcal{C}}^{\mathcal{G}}=-\boldsymbol{\omega}_{C}^{\times} \mathbf{D}_{\mathcal{C}}^{\mathcal{G}} \\
\dot{\mathbf{D}}_{\mathcal{T}}^{\mathcal{G}}=-\boldsymbol{\omega}_{T}^{\times} \mathbf{D}_{\mathcal{T}}^{\mathcal{G}} \\
\dot{\boldsymbol{\omega}}_{C}=\mathbf{J}_{C}^{-1}\left[\mathbf{r}_{C Q}^{\times} \mathbf{D}_{\mathcal{C}}^{\mathcal{G}} \mathbf{f}-\boldsymbol{\omega}_{C}^{\times} \mathbf{J}_{C} \boldsymbol{\omega}_{C}\right] \\
\dot{\boldsymbol{\omega}}_{T}=\mathbf{J}_{T}^{-1}\left[-\mathbf{r}_{T Q}^{\times} \mathbf{D}_{\mathcal{T}}^{\mathcal{G}} \mathbf{f}-\boldsymbol{\omega}_{T}^{\times} \mathbf{J}_{T} \boldsymbol{\omega}_{T}\right]
\end{gathered}
$$

such that

$$
\begin{gathered}
\mathbf{r}_{T Q}=\mathbf{D}_{\mathcal{T}}^{\mathcal{G}}\left[\mathbf{r}+\mathbf{D}_{\mathcal{G}}^{\mathcal{C}} \mathbf{r}_{C Q}\right] \\
\mathbf{f}=\left.f_{n} \mathbf{D}_{\mathcal{G}}^{\mathcal{N}}\left(\hat{\mathbf{n}}+\mu \hat{\mathbf{n}}_{\perp}\right)\right|_{t-h}
\end{gathered}
$$

where

$$
\begin{aligned}
& \mathbf{D}_{\mathcal{G}}^{\mathcal{N}}=\mathbf{D}_{\mathcal{G}}^{\mathcal{T}} \mathbf{D}_{\mathcal{T}}^{\mathcal{N}} \\
& \hat{\mathbf{n}}_{\perp}=\mathbf{D}_{\mathcal{N}}^{\mathcal{T}} \frac{\left(\mathbf{I}-\hat{\mathbf{n}}_{\mathcal{T}} \hat{\mathbf{n}}_{\mathcal{T}}^{T}\right) \dot{\mathbf{r}}_{S Q}}{\left\|\left(\mathbf{I}-\hat{\mathbf{n}}_{\mathcal{T}} \hat{\mathbf{n}}_{\mathcal{T}}^{T}\right) \dot{\mathbf{r}}_{S Q}\right\|} \\
& \mathbf{D}_{\mathcal{T}}^{\mathcal{N}}=\left[\begin{array}{ccc}
\cos \theta & 0 & -\sin \theta \\
-\sin \theta \sin \phi & \cos \phi & -\cos \theta \sin \phi \\
\sin \theta \cos \phi & \sin \phi & \cos \theta \cos \phi
\end{array}\right] \\
& \hat{\mathbf{n}}_{\mathcal{T}}=\mathbf{D}_{\mathcal{T}}^{\mathcal{N}} \hat{\mathbf{n}} \\
& \dot{\mathbf{r}}_{S Q}=-\boldsymbol{\omega}_{T}^{\times} \mathbf{r}_{T Q}+\mathbf{D}_{\mathcal{T}}^{\mathcal{G}}\left[\mathbf{v}+\mathbf{D}_{\mathcal{G}}^{\mathcal{C}} \boldsymbol{\omega}_{C}^{\times} \mathbf{r}_{C Q}\right] \\
& \tan \phi=-\frac{\hat{\mathbf{y}}_{\mathcal{T}}^{T} \mathbf{r}_{S Q}}{\hat{\mathbf{z}}_{\mathcal{T}}^{T} \mathbf{r}_{S Q}} \\
& \mathbf{r}_{S Q}=\mathbf{r}_{T Q}-\mathbf{r}_{T S}
\end{aligned}
$$

The compliance device based contact force model is summarized as

$$
\begin{aligned}
f_{n}= & -\left(1-a \frac{l_{0}}{l}\right)\left[\sum_{i=1}^{N} k_{i}\left(\hat{\mathbf{n}}_{\mathcal{C}}^{T} \hat{\mathbf{s}}_{i}\right)^{2}\right] d \\
& -a l_{0}\left(\frac{l_{0}}{l}-1\right)\left[\sum_{i=1}^{N} k_{i}\left(\hat{\mathbf{n}}_{\mathcal{C}}^{T} \hat{\mathbf{s}}_{i}\right)\left(\hat{\mathbf{x}}_{\mathcal{C}}^{T} \hat{\mathbf{s}}_{i}\right)\right]
\end{aligned}
$$

where

$$
\begin{gathered}
d=\mathbf{r}^{T} \mathbf{D}_{\mathcal{G}}^{\mathcal{N}} \hat{\mathbf{n}}+\mathbf{r}_{C Q}^{T} \mathbf{D}_{\mathcal{C}}^{\mathcal{N}} \hat{\mathbf{n}}-\mathbf{r}_{T S}^{T} \mathbf{D}_{\mathcal{T}}^{\mathcal{N}} \hat{\mathbf{n}} \\
l_{0}=\left\|\mathbf{r}_{C Q}-\mathbf{r}_{C R}\right\| \\
l=\left\|\mathbf{r}_{R Q}\right\|
\end{gathered}
$$




$$
\begin{gathered}
\hat{\mathbf{n}}_{\mathcal{C}}=\mathbf{D}_{\mathcal{C}}^{\mathcal{N}} \hat{\mathbf{n}} \\
\hat{\mathbf{s}}_{i}=\frac{\mathbf{r}_{R P}-\mathbf{r}_{R S_{i}}}{\left\|\mathbf{r}_{R P}-\mathbf{r}_{R S_{i}}\right\|} \\
\mathbf{D}_{\mathcal{C}}^{\mathcal{N}}=\mathbf{D}_{\mathcal{C}}^{\mathcal{G}} \mathbf{D}_{\mathcal{G}}^{\mathcal{T}} \mathbf{D}_{\mathcal{T}}^{\mathcal{N}} \\
\mathbf{r}_{R Q}=l_{0} \hat{\mathbf{x}}_{\mathcal{C}}-d \hat{\mathbf{n}}_{\mathcal{C}} \\
\mathbf{r}_{R P}=\left(1-a \frac{l_{0}}{l}\right) \mathbf{r}_{R Q}
\end{gathered}
$$

The constants $a, \mathbf{r}_{R S_{i}}, k_{i}, N$ and $\mu$ depend on the geometry and stiffness properties of the compliance device and the friction properties of the nozzle/probe interaction surface material, respectively, and $\mathbf{r}_{C Q}, \mathbf{r}_{C R}$ and $\mathbf{r}_{T S}$ on the geometry of the Chaser and Target. The constant vectors are given by

$$
\begin{gathered}
\hat{\mathbf{n}}=\{0,0,1\}^{T} ; \quad \hat{\mathbf{x}}_{\mathcal{C}}=\{1,0,0\}^{T} ; \\
\hat{\mathbf{y}}_{\mathcal{T}}=\{0,1,0\}^{T} ; \quad \hat{\mathbf{z}}_{\mathcal{T}}=\{0,0,1\}^{T} ;
\end{gathered}
$$

In contrast to the system model in Eqs. (1)-(8), the above model has a state vector of dimension 30. The reduced representation of the translational motion states using the relative motion states only does not impair the generality of the dynamic analysis that will follow. Indeed, since the contact force is an internal force, the missing states, that is the mass barycenter position and velocity, are determined from the initial conditions.

\section{Design Model}

A design model is developed below by using a simpler contact dynamics model and by linearizing the state-space equations around nominal pre-contact values under the assumptions of small linear and angular displacements. Earlier efforts on modeling the contact dynamics have made the spring-dashpot system a widely used model because of its linearity ${ }^{17}$. In the present section, a spring-dashpot contact force model is considered for the linearization of the statespace model.

\section{Simpler contact dynamics model}

Assumption-6: The normal force is modeled in accordance with a spring-dashpot model ${ }^{10,17,27}$, where the stiffness and damping coefficients are scalar time-invariant parameters, that is

$$
f_{n}=-k d-b \dot{d}
$$

where $k$ and $b$ denote the stiffness and the damping coefficients, respectively.

The penetration depth, penetration rate and other state dependent variables are derived from the previous section (see Appendix-A)

$$
\begin{gathered}
d=\mathbf{r}^{T} \mathbf{D}_{\mathcal{G}}^{\mathcal{N}} \hat{\mathbf{n}}+\mathbf{r}_{C Q}^{T} \mathbf{D}_{\mathcal{C}}^{\mathcal{N}} \hat{\mathbf{n}}-\mathbf{r}_{T S}^{T} \mathbf{D}_{\mathcal{T}}^{\mathcal{N}} \hat{\mathbf{n}} \\
\dot{d}=\mathbf{v}^{T} \mathbf{D}_{\mathcal{G}}^{\mathcal{N}} \hat{\mathbf{n}}+\mathbf{r}^{T} \dot{\mathbf{D}}_{\mathcal{G}}^{\mathcal{N}} \hat{\mathbf{n}}+\mathbf{r}_{C Q}^{T} \dot{\mathbf{D}}_{\mathcal{C}}^{\mathcal{N}} \hat{\mathbf{n}}-\mathbf{r}_{T S}^{T} \dot{\mathbf{D}}_{\mathcal{T}}^{\mathcal{N}} \hat{\mathbf{n}}
\end{gathered}
$$

where

$$
\begin{gathered}
\mathbf{D}_{\mathcal{C}}^{\mathcal{N}}=\mathbf{D}_{\mathcal{C}}^{\mathcal{G}} \mathbf{D}_{\mathcal{G}}^{\mathcal{T}} \mathbf{D}_{\mathcal{T}}^{\mathcal{N}} \\
\dot{\mathbf{D}}_{\mathcal{T}}^{\mathcal{N}}=\boldsymbol{\omega}_{N}^{\times} \mathbf{D}_{\mathcal{T}}^{\mathcal{N}} \\
\dot{\mathbf{D}}_{\mathcal{G}}^{\mathcal{N}}=\mathbf{D}_{\mathcal{G}}^{\mathcal{T}}\left[\boldsymbol{\omega}_{T}+\boldsymbol{\omega}_{N}\right]^{\times} \mathbf{D}_{\mathcal{T}}^{\mathcal{N}} \\
\dot{\mathbf{D}}_{\mathcal{C}}^{\mathcal{N}}=-\boldsymbol{\omega}_{C}^{\times} \mathbf{D}_{\mathcal{C}}^{\mathcal{N}}+\mathbf{D}_{\mathcal{C}}^{\mathcal{G}} \mathbf{D}_{\mathcal{G}}^{\mathcal{T}}\left[\boldsymbol{\omega}_{T}+\boldsymbol{\omega}_{N}\right]^{\times} \mathbf{D}_{\mathcal{T}}^{\mathcal{N}} \\
\boldsymbol{\omega}_{N}=\{\dot{\phi}, 0,0\}^{T} \\
\dot{\phi}=\frac{\left[\hat{\mathbf{y}}_{\mathcal{T}}^{T}+\hat{\mathbf{z}}_{\mathcal{T}}^{T} \tan \phi\right] \dot{\mathbf{r}}_{S Q}}{\left[\hat{\mathbf{y}}_{\mathcal{T}}^{T} \tan \phi-\hat{\mathbf{z}}_{\mathcal{T}}^{T}\right] \mathbf{r}_{S Q}}
\end{gathered}
$$

where $\phi, \mathbf{r}_{S Q}$ and $\dot{\mathbf{r}}_{S Q}$ are obtained from Eqs. (56)-(58). The Chaser-Target dynamics in Eqs. (44)-(58) along with the spring-dashpot contact dynamics in Eqs. (68)-(76) constitute the spacecraft system dynamics model.

Assumption-7: The nozzle surface is frictionless

Assumption-7 simplifies the model by eliminating the nonlinearity present in the system due to Coulomb friction model. The contact force is therefore rewritten from Eq. (51) as

$$
\mathbf{f}=f_{n}(t-h) \hat{\mathbf{n}}_{\mathcal{T}}^{*}
$$

\section{Linearization}

The envisioned scenario consists of the Chaser having reached a zero relative attitude rate with respect to the Target and moving towards it at a very small speed with the probe aligned along the $-x$-axis of the Target and with identical $z$-axes in the Chaser and Target frames. For simplicity, the Target and inertial frames are assumed to coincide prior to contact.

Nominal state: Let the nominal state be such that the Chaser and Target bodies are at rest, and the Chaser position is $\mathbf{r}_{\circ}$ with respect to the Target. The attitude of the Target frame $\mathcal{T}$ at the nominal state is assumed to be along the inertial frame $\mathcal{G}$, and that of the Chaser frame $\mathcal{C}$ is such that $z_{\mathcal{C}}$-axis is along $z_{\mathcal{G}}$-axis and $x_{\mathcal{C}}$-axis is opposite to $x_{\mathcal{G}}$-axis

$$
\begin{gathered}
\mathbf{r}^{*}=\mathbf{r}_{\circ} \\
\mathbf{v}^{*}=\mathbf{0} \\
\mathbf{D}_{\mathcal{C}}^{\mathcal{G} *}=\left[\begin{array}{ccc}
-1 & 0 & 0 \\
0 & -1 & 0 \\
0 & 0 & 1
\end{array}\right]=\mathbf{I}_{1}
\end{gathered}
$$




$$
\begin{gathered}
\mathbf{D}_{\mathcal{T}}^{\mathcal{G} *}=\left[\begin{array}{ccc}
1 & 0 & 0 \\
0 & 1 & 0 \\
0 & 0 & 1
\end{array}\right]=\mathbf{I} \\
\boldsymbol{\omega}_{C}^{*}=\mathbf{0} \\
\boldsymbol{\omega}_{T}^{*}=\mathbf{0}
\end{gathered}
$$

It is to be noted that the penetration depth at the nominal state depends on the choice of $\mathbf{r}_{\circ}$. Considering the penetration depth at the nominal state to be zero, it follows from Eq. (32)

$$
d^{*}=\mathbf{r}_{S Q}^{* T} \mathbf{D}_{\mathcal{T}}^{\mathcal{N} *} \hat{\mathbf{n}}=\hat{\mathbf{n}}_{\mathcal{T}}^{* T} \mathbf{r}_{S Q}^{*}=0
$$

where

$$
\hat{\mathbf{n}}_{\mathcal{T}}^{*}=\mathbf{D}_{\mathcal{T}}^{\mathcal{N} *} \hat{\mathbf{n}}
$$

is the local normal unit vector at the nominal state expressed in the Target frame. Let the nominal state vector $\mathbf{r}_{S Q}^{*}$ be denoted by $\mathbf{p}^{*}$, then using Eq. (31) yields

$$
\begin{aligned}
\mathbf{p}^{*} & =\mathbf{D}_{\mathcal{T}}^{\mathcal{G} *}\left[\mathbf{r}_{\circ}+\mathbf{D}_{\mathcal{G}}^{\mathcal{C} *} \mathbf{r}_{C Q}\right]-\mathbf{r}_{T S} \\
& =\mathbf{r}_{\circ}+\mathbf{I}_{1} \mathbf{r}_{C Q}-\mathbf{r}_{T S}
\end{aligned}
$$

where $\mathbf{D}_{\mathcal{T}}^{\mathcal{G} *}$ and $\mathbf{D}_{\mathcal{G}}^{\mathcal{C} *}$ are substituted from Eqs. (81) and (80), respectively. Rewriting the penetration depth and azimuthal angle at the nominal state in terms of $\mathbf{p}^{*}$

$$
\begin{gathered}
d^{*}=\hat{\mathbf{n}}_{\mathcal{T}}^{* T} \mathbf{p}^{*}=0 \\
\tan \phi^{*}=-\frac{\hat{\mathbf{y}}_{\mathcal{T}}^{T} \mathbf{p}^{*}}{\hat{\mathbf{z}}_{\mathcal{T}}^{T} \mathbf{p}^{*}}
\end{gathered}
$$

where Eq. (88) is a direct result of Eq. (30) at the nominal state. The direction cosine matrix from the Nozzle frame to the Target frame at the nominal state

$$
\mathbf{D}_{\mathcal{T}}^{\mathcal{N} *}=\left[\begin{array}{ccc}
\cos \theta & 0 & -\sin \theta \\
-\sin \theta \sin \phi^{*} & \cos \phi^{*} & -\cos \theta \sin \phi^{*} \\
\sin \theta \cos \phi^{*} & \sin \phi^{*} & \cos \theta \cos \phi^{*}
\end{array}\right]
$$

can be found by substituting the resulting $\phi^{*}$ from Eq. (88).

Assumption-8: The perturbations in the state are small with respect to the nominal state.

Small perturbations: Let the small perturbations in the system at the above assumed nominal states be denoted by $\boldsymbol{\delta} \boldsymbol{r}, \boldsymbol{\delta} \boldsymbol{v}, \boldsymbol{\delta} \gamma_{C}, \delta \gamma_{T}, \delta \boldsymbol{\omega}_{C}$ and $\delta \boldsymbol{\omega}_{T}$ for the relative position, the relative velocity, the Chaser and Target attitudes, and the Chaser and Target angular velocities, respectively. The
Chaser and Target attitudes are defined as

$$
\boldsymbol{\delta} \boldsymbol{\gamma}_{C}=\left\{\begin{array}{l}
\delta \phi_{C} \\
\delta \theta_{C} \\
\delta \psi_{C}
\end{array}\right\} ; \quad \boldsymbol{\delta} \boldsymbol{\gamma}_{T}=\left\{\begin{array}{l}
\delta \phi_{T} \\
\delta \theta_{T} \\
\delta \psi_{T}
\end{array}\right\}
$$

where $\delta \phi, \delta \theta$ and $\delta \psi$ denote the angular perturbations about the $x, y$ and $z$ axes, respectively. Therefore, the angular kinematic equations in (46) and (47) reduce to

$$
\delta \dot{\gamma}_{C}=\delta \omega_{C} ; \quad \delta \dot{\gamma}_{T}=\delta \omega_{T}
$$

Perturbations in the direction cosine matrices can be written as

$$
\begin{aligned}
\boldsymbol{\delta} \mathbf{D}_{\mathcal{C}}^{\mathcal{G}}= & {\left[\begin{array}{ccc}
0 & -\delta \psi_{C} & -\delta \theta_{C} \\
\delta \psi_{C} & 0 & \delta \phi_{C} \\
-\delta \theta_{C} & \delta \phi_{C} & 0
\end{array}\right]=-\boldsymbol{\delta} \boldsymbol{\gamma}_{C}^{\times} \mathbf{I}_{1} } \\
\boldsymbol{\delta} \mathbf{D}_{\mathcal{T}}^{\mathcal{G}}= & {\left[\begin{array}{ccc}
0 & \delta \psi_{T} & -\delta \theta_{T} \\
-\delta \psi_{T} & 0 & \delta \phi_{T} \\
\delta \theta_{T} & -\delta \phi_{T} & 0
\end{array}\right]=-\boldsymbol{\delta} \boldsymbol{\gamma}_{T}^{\times} }
\end{aligned}
$$

where the superscript $\times$ denotes the cross product operator as provided in Eq. (10). The perturbations in $\mathbf{r}_{S Q}$ and $\dot{\mathbf{r}}_{S Q}$ are found using Eqs. (31)

$$
\begin{aligned}
\boldsymbol{\delta} \mathbf{r}_{S Q} & =\mathbf{D}_{\mathcal{T}}^{\mathcal{G} *}\left[\boldsymbol{\delta} \mathbf{r}+\boldsymbol{\delta} \mathbf{D}_{\mathcal{G}}^{\mathcal{C}} \mathbf{r}_{C Q}\right]+\boldsymbol{\delta} \mathbf{D}_{\mathcal{T}}^{\mathcal{G}}\left[\mathbf{r}_{\circ}+\mathbf{D}_{\mathcal{G}}^{\mathcal{C} *} \mathbf{r}_{C Q}\right] \\
& =\boldsymbol{\delta} \mathbf{r}+\mathbf{P}_{C} \boldsymbol{\delta} \gamma_{C}+\mathbf{P}_{T} \boldsymbol{\delta} \boldsymbol{\gamma}_{T} \\
\boldsymbol{\delta} \dot{\mathbf{r}}_{S Q} & =\boldsymbol{\delta} \mathbf{v}+\mathbf{P}_{C} \boldsymbol{\delta} \boldsymbol{\omega}_{C}+\mathbf{P}_{T} \boldsymbol{\delta} \boldsymbol{\omega}_{T}
\end{aligned}
$$

where

$$
\mathbf{P}_{C}=-\mathbf{I}_{1} \mathbf{r}_{C Q}^{\times} ; \quad \mathbf{P}_{T}=\left[\mathbf{r}_{\circ}+\mathbf{I}_{1} \mathbf{r}_{C Q}\right]^{\times}
$$

The perturbation in angle $\phi$ is found from Eqs. (30) as

$$
\begin{aligned}
\delta \phi & =\frac{\left[\hat{\mathbf{y}}_{\mathcal{T}}^{T}+\hat{\mathbf{z}}_{\mathcal{T}}^{T} \tan \phi^{*}\right] \delta \mathbf{r}_{S Q}}{\left[\hat{\mathbf{y}}_{\mathcal{T}}^{T} \tan \phi^{*}-\hat{\mathbf{z}}_{\mathcal{T}}^{T}\right] \mathbf{r}_{S Q}^{*}} \\
& =\frac{-\mathbf{p}^{* T} \hat{\mathbf{x}}_{\mathcal{T}}^{\times}}{\mathbf{p}^{* T}\left[\mathbf{I}-\hat{\mathbf{x}}_{\mathcal{T}} \hat{\mathbf{x}}_{\mathcal{T}}^{T}\right] \mathbf{p}^{*}} \boldsymbol{\delta} \mathbf{r}_{S Q}
\end{aligned}
$$

It can be observed from Fig. (3) that

$$
\begin{gathered}
\mathbf{p}^{* T} \hat{\mathbf{x}}_{\mathcal{T}}^{\times}=\left\|\mathbf{p}^{*}\right\| \sin \theta \hat{\mathbf{t}}_{\mathcal{T}}^{* T} \\
\mathbf{p}^{* T} \hat{\mathbf{x}}_{\mathcal{T}}=\left\|\mathbf{p}^{*}\right\| \cos \theta
\end{gathered}
$$

where $\hat{\mathbf{t}}_{\mathcal{T}}^{*}=\mathbf{D}_{\mathcal{T}}^{\mathcal{N}} * \hat{\mathbf{t}}$ is the tangential unit vector at the contact point at nominal state expressed in Target frame. Substituting from Eqs. (98) and (99) in Eq. (97) yields

$$
\delta \phi=p^{*} \hat{\mathbf{t}}_{\mathcal{T}}^{* T} \delta \mathbf{r}_{S Q}
$$


where

$$
p^{*}=\frac{-1}{\left\|\mathbf{p}^{*}\right\| \sin \theta}
$$

The perturbations in $\mathbf{D}_{\mathcal{T}}^{\mathcal{N}}$ about the nominal state can be found from Eq. (11) as

$$
\begin{aligned}
\delta \mathbf{D}_{\mathcal{T}}^{\mathcal{N}} & =\delta \phi\left[\begin{array}{ccc}
0 & 0 & 0 \\
-\sin \theta \cos \phi^{*} & -\sin \phi^{*} & -\cos \theta \cos \phi^{*} \\
-\sin \theta \sin \phi^{*} & \cos \phi^{*} & -\cos \theta \sin \phi^{*}
\end{array}\right] \\
& =\delta \phi \mathbf{x}_{\mathcal{T}}^{\times} \mathbf{D}_{\mathcal{T}}^{\mathcal{N}}{ }^{*}
\end{aligned}
$$

The right hand side of Eq. (102) clearly expresses that the perturbation in the attitude of the Nozzle frame with respect to the Target frame is a rotation of the Nozzle frame by a small angle $\delta \phi$ about the axis $x_{\mathcal{T}}$. Using Eq. (32), the perturbation in the penetration depth is found as

$$
\begin{aligned}
\delta d & =\mathbf{p}^{* T} \boldsymbol{\delta} \hat{\mathbf{n}}_{\mathcal{T}}+\hat{\mathbf{n}}_{\mathcal{T}}^{* T} \boldsymbol{\delta} \mathbf{r}_{S Q} \\
& =\mathbf{p}^{* T} \boldsymbol{\delta} \mathbf{D}_{\mathcal{T}}^{\mathcal{N}} \hat{\mathbf{n}}+\hat{\mathbf{n}}_{\mathcal{T}}^{* T} \boldsymbol{\delta} \mathbf{r}_{S Q}
\end{aligned}
$$

and substituting $\delta \mathbf{D}_{\mathcal{T}}^{\mathcal{N}}$ from Eq. (102) in Eq. (103) gives

$$
\begin{aligned}
\delta d & =\delta \phi \mathbf{p}^{* T} \mathbf{x}_{\mathcal{T}}^{\times} \hat{\mathbf{n}}_{\mathcal{T}}^{*}+\hat{\mathbf{n}}_{\mathcal{T}}^{* T} \boldsymbol{\delta} \mathbf{r}_{S Q} \\
& =\hat{\mathbf{n}}_{\mathcal{T}}^{* T} \boldsymbol{\delta} \mathbf{r}_{S Q}
\end{aligned}
$$

Equation (104) follows from the choice of the Nozzle frame, that the unit vectors $\mathbf{p}^{*}, \mathbf{x}_{\mathcal{T}}$ and $\hat{\mathbf{n}}_{\mathcal{T}}^{*}$ are coplanar, and hence the box product $\mathbf{p}^{* T} \mathbf{x}_{\mathcal{T}}^{\times} \hat{\mathbf{n}}_{\mathcal{T}}^{*}=0$. The penetration rate is written similarly as

$$
\delta \dot{d}=\hat{\mathbf{n}}_{\mathcal{T}}^{* T} \boldsymbol{\delta} \dot{\mathbf{r}}_{S Q}
$$

Full order Design model: On substituting Eqs. (94)-(77) in the nonlinear model, the linearized equations of motion results in

$$
\begin{gathered}
\boldsymbol{\delta} \dot{\mathbf{r}}=\boldsymbol{\delta} \mathbf{v} \\
\boldsymbol{\delta} \dot{\gamma}_{C}=\boldsymbol{\delta} \boldsymbol{\omega}_{C} \\
\boldsymbol{\delta} \dot{\gamma}_{T}=\boldsymbol{\delta} \boldsymbol{\omega}_{T} \\
\boldsymbol{\delta} \dot{\mathbf{v}}=\frac{f_{n}(t-h)}{m_{r}} \hat{\mathbf{n}}_{\mathcal{T}}^{*} \\
\delta \dot{\boldsymbol{\omega}}_{C}=f_{n}(t-h) \mathbf{J}_{C}^{-1} \mathbf{P}_{C}^{T} \hat{\mathbf{n}}_{\mathcal{T}}^{*} \\
\delta \dot{\boldsymbol{\omega}}_{T}=f_{n}(t-h) \mathbf{J}_{T}^{-1}\left(\mathbf{P}_{T}^{T}+\mathbf{r}_{T S}^{\times}\right) \hat{\mathbf{n}}_{\mathcal{T}}^{*}
\end{gathered}
$$

where

$$
\begin{gathered}
f_{n}=-k \delta d-b \delta \dot{d} \\
\delta d=\hat{\mathbf{n}}_{\mathcal{T}}^{* T} \boldsymbol{\delta} \mathbf{r}_{S Q} \\
\delta \dot{d}=\hat{\mathbf{n}}_{\mathcal{T}}^{* T} \boldsymbol{\delta} \dot{\mathbf{r}}_{S Q}
\end{gathered}
$$

$$
\begin{gathered}
\delta \mathbf{r}_{S Q}=\boldsymbol{\delta} \mathbf{r}+\mathbf{P}_{C} \boldsymbol{\delta} \boldsymbol{\gamma}_{C}+\mathbf{P}_{T} \boldsymbol{\delta} \boldsymbol{\gamma}_{T} \\
\delta \dot{\mathbf{r}}_{S Q}=\boldsymbol{\delta} \mathbf{v}+\mathbf{P}_{C} \boldsymbol{\delta} \boldsymbol{\omega}_{C}+\mathbf{P}_{T} \boldsymbol{\delta} \boldsymbol{\omega}_{T}
\end{gathered}
$$

where Eq. (112) takes into account the nominal state $d^{*}=0$ as provided in Eq. (84). The constant matrices $\mathbf{P}_{C}$ and $\mathbf{P}_{T}$ are provided in Eq. (96).

The assumption of small perturbations in the attitude of the system led to consider a three parameter attitude representation, namely $\delta \phi, \delta \theta$ and $\delta \psi$ about $x, y$ and $z$ axes, respectively for each Chaser and Target body. The linearized system model given by Eqs. (106)-(111), thus, resulted in a system of order 18 . However, a redundancy can still be noticed in the full order model.

Reduced order Design model: A reduced order model allows an in-depth analysis of a system and lowers the computational effort of the simulation. As for the problem at hand, it will be shown at the end of the section that a reduced order model leads to a simplified system to proceed with the stability analysis.

It is observed from the state equations in Eqs. (109)-(114) that the perturbation vector $\delta \mathbf{r}_{S Q}$ readily defines a set of state variables. In the rest of the section, the focus is made on the contact point dynamics, since a stability of the former corresponds to the stability in the spacecraft dynamics (see Appendix-B). The dynamics of the contact point follows from Eqs. (109)-(116)

$$
\delta \ddot{\mathbf{r}}_{S Q}(t)=-\mathbf{B} \boldsymbol{\delta} \dot{\mathbf{r}}_{S Q}(t-h)-\mathbf{K} \boldsymbol{\delta} \mathbf{r}_{S Q}(t-h)
$$

where

$$
\begin{gathered}
\mathbf{B}=b \mathbf{M} \hat{\mathbf{n}}_{\mathcal{T}}^{*} \hat{\mathbf{n}}_{\mathcal{T}}^{* T} \\
\mathbf{K}=k \mathbf{M} \hat{\mathbf{n}}_{\mathcal{T}}^{*} \hat{\mathbf{n}}_{\mathcal{T}}^{* T} \\
\mathbf{M}=\frac{\mathbf{I}}{m_{r}}+\mathbf{P}_{C} \mathbf{J}_{C}^{-1} \mathbf{P}_{C}^{T}+\mathbf{P}_{T} \mathbf{J}_{T}^{-1}\left(\mathbf{P}_{T}^{T}+\mathbf{r}_{T S}^{\times}\right)
\end{gathered}
$$

On comparing the system described by Eqs. (106)-(116) and Eq. (117), it can be stated that the number of state variables has been reduced from 18 to 6 .

Penetration depth dynamics: The penetration depth at point $Q$ is along the normal to the nozzle surface, given by Eq. (113). Differentiating Eq. (113) twice yields

$$
\delta \ddot{d}=\hat{\mathbf{n}}_{\mathcal{T}}^{* T} \boldsymbol{\delta} \ddot{\mathbf{r}}_{S Q}
$$

and substituting from Eq. (117) leads to

$$
\delta \ddot{d}=-\hat{\mathbf{n}}_{\mathcal{T}}^{* T} \mathbf{B} \boldsymbol{\delta} \dot{\mathbf{r}}_{S Q}(t-h)-\hat{\mathbf{n}}_{\mathcal{T}}^{* T} \mathbf{K} \boldsymbol{\delta} \mathbf{r}_{S Q}(t-h)
$$


It can be noticed from Eqs. (118) and (119)

$$
\begin{aligned}
& \hat{\mathbf{n}}_{\mathcal{T}}^{* T} \mathbf{B}=b_{d} \hat{\mathbf{n}}_{\mathcal{T}}^{* T} \\
& \hat{\mathbf{n}}_{\mathcal{T}}^{* T} \mathbf{K}=k_{d} \hat{\mathbf{n}}_{\mathcal{T}}^{* T}
\end{aligned}
$$

where

$$
\begin{aligned}
b_{d} & =b \hat{\mathbf{n}}_{\mathcal{T}}^{* T} \mathbf{M} \hat{\mathbf{n}}_{\mathcal{T}}^{*} \\
k_{d} & =k \hat{\mathbf{n}}_{\mathcal{T}}^{* T} \mathbf{M} \hat{\mathbf{n}}_{\mathcal{T}}^{*}
\end{aligned}
$$

Therefore, it follows from Eqs. (113), (114), (122) and (123)

$$
\delta \ddot{d}=-b_{d} \delta \dot{d}(t-h)-k_{d} \delta d(t-h)
$$

Contact point dynamics: Along with the direction normal to the nozzle surface $\hat{\mathbf{n}}$, the radial direction $\hat{\mathbf{m}}$ and tangential direction $\hat{\mathbf{t}}$ constitute the Nozzle frame, such that

$$
\begin{aligned}
\hat{\mathbf{m}} & =\mathbf{x}_{\mathcal{N}} \\
\hat{\mathbf{t}} & =\mathbf{y}_{\mathcal{N}}
\end{aligned}
$$

In this section the radial and tangential motion of point $Q$ is considered along the surface of the nozzle.

\section{Dynamics along the radial direction}

The radial component of position of point $Q$ on the nozzle surface can be written as

$$
m=\hat{\mathbf{m}}_{\mathcal{T}}^{T} \mathbf{r}_{S Q}
$$

where, $\hat{\mathbf{m}}_{\mathcal{T}}=\mathbf{D}_{\mathcal{T}}^{\mathcal{N}} \hat{\mathbf{m}}$. A small perturbation in the radial position can be written as

$$
\delta m=\hat{\mathbf{m}}_{\mathcal{T}}^{* T} \boldsymbol{\delta} \mathbf{r}_{S Q}+\mathbf{r}_{S Q}^{* T} \boldsymbol{\delta} \mathbf{D}_{\mathcal{T}}^{\mathcal{N}} \hat{\mathbf{m}}
$$

where

$$
\hat{\mathbf{m}}_{\mathcal{T}}^{*}=\mathbf{D}_{\mathcal{T}}^{\mathcal{N}}{ }^{*} \hat{\mathbf{m}}=\left\{\begin{array}{c}
\cos \theta \\
-\sin \theta \sin \phi^{*} \\
\sin \theta \cos \phi^{*}
\end{array}\right\}
$$

On substituting Eq. (102) in Eq. (129)

$$
\begin{aligned}
\delta m & =\hat{\mathbf{m}}_{\mathcal{T}}^{* T} \boldsymbol{\delta} \mathbf{r}_{S Q}+\delta \phi \mathbf{p}^{* T} \mathbf{x}_{\mathcal{T}}^{\times} \hat{\mathbf{m}}_{\mathcal{T}}^{*} \\
& =\hat{\mathbf{m}}_{\mathcal{T}}^{* T} \boldsymbol{\delta} \mathbf{r}_{S Q}
\end{aligned}
$$

Equation (131) follows from the observation that $\mathbf{p}^{* T} \mathbf{x}_{\mathcal{T}}^{\times} \hat{\mathbf{m}}_{\mathcal{T}}^{*}=0$, as $\mathbf{p}^{*}$ is along $\hat{\mathbf{m}}_{\mathcal{T}}^{*}$. Differentiating Eq. (131) twice and substituting $\delta \ddot{\mathbf{r}}_{S Q}, \boldsymbol{\delta} \dot{\mathbf{r}}_{S Q}$ and $\delta \mathbf{r}_{S Q}$ from Eqs. (117), (113) and (114), respectively, yields

$$
\delta \ddot{m}=-b_{m} \delta \dot{d}(t-h)-k_{m} \delta d(t-h)
$$

where

$$
b_{m}=b \hat{\mathbf{m}}_{\mathcal{T}}^{* T} \mathbf{M} \hat{\mathbf{n}}_{\mathcal{T}}^{*}
$$

$$
k_{m}=k \hat{\mathbf{m}}_{\mathcal{T}}^{* T} \mathbf{M} \hat{\mathbf{n}}_{\mathcal{T}}^{*}
$$

\section{Dynamics along the tangential direction}

The tangential component of the position vector of point $Q$ is characterized by the angle $\delta \phi$ given in Eq. (100) as

$$
\delta \phi=p^{*} \hat{\mathbf{t}}_{\mathcal{T}}^{* T} \boldsymbol{\delta} \mathbf{r}_{S Q}
$$

Similarly, differentiating Eq. (135) twice and substituting $\delta \ddot{\mathbf{r}}_{S Q}, \delta \dot{\mathbf{r}}_{S Q}$ and $\boldsymbol{\delta} \mathbf{r}_{S Q}$ from Eqs. (117), (113) and (114), respectively, gives

$$
\delta \ddot{\phi}=-b_{t} \delta \dot{d}(t-h)-k_{t} \delta d(t-h)
$$

where

$$
\begin{aligned}
b_{t} & =b p^{*} \hat{\mathbf{t}}_{\mathcal{T}}^{* T} \mathbf{M} \hat{\mathbf{n}}_{\mathcal{T}}^{*} \\
k_{t} & =k p^{*} \hat{\mathbf{t}}_{\mathcal{T}}^{* T} \mathbf{M} \hat{\mathbf{n}}_{\mathcal{T}}^{*}
\end{aligned}
$$

Summary: It can be observed from Eqs. (113), (131) and (135) that the perturbations along the normal, the radial and the tangential directions are linear combinations of the vector $\delta \mathbf{r}_{S Q}$, such that

$$
\left\{\begin{array}{c}
\delta m \\
\delta \phi \\
\delta d
\end{array}\right\}=\left[\begin{array}{c}
\hat{\mathbf{m}}_{\mathcal{T}}^{* T} \\
p^{*} \hat{\mathbf{t}}_{\mathcal{T}}^{* T} \\
\hat{\mathbf{n}}_{\mathcal{T}}^{* T}
\end{array}\right] \delta \mathbf{r}_{S Q}
$$

where $\hat{\mathbf{m}}_{\mathcal{T}}^{*}, \hat{\mathbf{t}}_{\mathcal{T}}^{*}$ and $\hat{\mathbf{n}}_{\mathcal{T}}^{*}$ are the columns of the matrix $\mathbf{D}_{\mathcal{T}}^{\mathcal{N}}$. The differential equations along the three directions are summarized as

$$
\begin{aligned}
\delta \ddot{d} & =-b_{d} \delta \dot{d}(t-h)-k_{d} \delta d(t-h) \\
\delta \ddot{m} & =-b_{m} \delta \dot{d}(t-h)-k_{m} \delta d(t-h) \\
\delta \ddot{\phi} & =-b_{t} \delta \dot{d}(t-h)-k_{t} \delta d(t-h)
\end{aligned}
$$

where $b_{d}, k_{d}, b_{m}, k_{m}, b_{t}$ and $k_{t}$ are given by Eqs. (124), (125), (133), (134), (137) and (138), respectively. It can be noticed from Eqs. (140)-(142) that the dynamics of the contact point normal to the nozzle surface is decoupled from that along the radial and tangential directions. A perturbation along the normal direction leads to a subsequent motion of the contact point along the radial and tangential directions, moreover, a stability in the motion along the normal direction implies a stability in the radial and tangential directions. 


\section{Stability Analysis}

\section{Pole placement method}

The differential equations (140)-(142) are transformed to the Laplace domain as

$$
s^{2} \mathbf{x}(s)+s \mathbf{B}_{\mathbf{x}} \mathbf{x}(s)+\mathbf{K}_{\mathbf{x}} \mathbf{x}(s)=\mathbf{0}
$$

where $\mathbf{x}(s)=\{\delta d, \delta m, \delta \phi\}^{T}$ is the reduced state in Laplace domain, and $\mathbf{B}_{\mathbf{x}}=e^{-s h}\left[\begin{array}{ccc}b_{d} & 0 & 0 \\ b_{m} & 0 & 0 \\ b_{t} & 0 & 0\end{array}\right]$ and $\mathbf{K}_{\mathbf{x}}=$ $e^{-s h}\left[\begin{array}{ccc}k_{d} & 0 & 0 \\ k_{m} & 0 & 0 \\ k_{t} & 0 & 0\end{array}\right]$ are the damping and stiffness matrices, respectively. The characteristic equation results from Eq. (143) as follows

$$
s^{4}\left(s^{2}+e^{-s h} b_{d} s+e^{-s h} k_{d}\right)=0
$$

The $6^{\text {th }}$ order characteristic equation shows that the modes of the system are associated with the dynamics of the contact point along the normal direction. In the remaining section, only the dynamics of the penetration depth is considered, which is responsible for the stability of the system. Before proceeding to the validation of the stability analysis, a few simplifying assumptions are made on the geometry of the Chaser and Target bodies with no loss of generality.

Assumption-9: The center of mass of the Target coincides with the origin of the Nozzle frame. The probe is along the $x$-axis of the Chaser body. The axes of the Chaser and Target frames coincide with the principal axes of inertia of the Chaser and Target rigid bodies, respectively.

As a result

$$
\mathbf{r}_{T S}=\mathbf{0} ; \quad \mathbf{r}_{C Q}=\left\{l_{0}, 0,0\right\}^{T}
$$

where $l_{0}$ is the length of the probe. Let the moment of inertia of Chaser and Target bodies be

$$
\mathbf{J}_{C}=\left[\begin{array}{ccc}
J_{C_{x}} & 0 & 0 \\
0 & J_{C_{y}} & 0 \\
0 & 0 & J_{C_{z}}
\end{array}\right] ; \mathbf{J}_{T}=\left[\begin{array}{ccc}
J_{T_{x}} & 0 & 0 \\
0 & J_{T_{y}} & 0 \\
0 & 0 & J_{T_{z}}
\end{array}\right]
$$

where $J_{C_{x}}, J_{C_{y}}, J_{C_{z}}, J_{T_{x}}, J_{T_{y}}$ and $J_{T_{z}}$ are the principal components of moment of inertia of Chaser and Target, respectively. Using these simplifications, $b_{d}$ and $k_{d}$ from Eqs. (124) and (125) result in

$$
\begin{aligned}
b_{d} & =\frac{b}{m^{\dagger}} \\
k_{d} & =\frac{k}{m^{\dagger}}
\end{aligned}
$$

$$
\begin{aligned}
\frac{1}{m^{\dagger}}=\frac{1}{m_{r}} & +\cos ^{2} \phi^{*}\left(\frac{l_{0}^{2} \cos ^{2} \theta}{J_{C_{y}}}+\frac{m^{* 2}}{J_{T_{y}}}\right) \\
& +\sin ^{2} \phi^{*}\left(\frac{l_{0}^{2} \cos ^{2} \theta}{J_{C_{z}}}+\frac{m^{* 2}}{J_{T_{z}}}\right)
\end{aligned}
$$

where $\phi^{*}$ and $m^{*}$ denote the nominal azimuthal angle of the point of contact and the nominal distance $S Q$. Equations (147)-(149) encompasses the 2D modeling result by Zebenay et $a l .{ }^{28}$. The latter is retrieved from Eqs. (147)-(149) by assuming the motion confined to the common $x z$-plane of the Chaser and Target $\left(\phi^{*}=0\right)$, and the Target inertially fixed $\left(J_{T_{y}} \rightarrow \infty\right)$. It is evident from Eqs. (147)-(149) that the effective damping, $b_{d}$ and the effective stiffness, $k_{d}$ increase as the point of contact moves closer to the nozzle tip. Further, $b_{d}$ and $k_{d}$ also increase with an increase in the probe length and a decrease in the semi-cone angle of the nozzle.

In order to determine the stability region in presence of the delay $h$, the roots of the characteristic equation in Eq. (144) must lie on the left half complex plane. The stability analysis follows the pole placement method for linear timedelay systems of the second order as described in Marshall et al. (1992) ${ }^{29}$. Let $h$ and $\omega$ denote the delay value and the value of the first crossing frequency of the poles into the right half complex plane, then the following relations hold

$$
\begin{aligned}
& \omega=\sqrt{\frac{b_{d}^{2}}{2}+\sqrt{\frac{b_{d}^{4}}{4}+k_{d}^{2}}} \\
& h=\frac{1}{\omega} \tan ^{-1}\left(\frac{\omega b_{d}}{k_{d}}\right)
\end{aligned}
$$

Equations (150) and (151) give the variation of the effective damping, $b_{d}$, and the effective stiffness, $k_{d}$, with respect to the time delay, $h$, such that the system in Eq. (143) is marginally stable (has poles with zero real part).

\section{Numerical example}

The stability regions are determined using pole placement method as an example case for a typical Chaser and Target body interacting at one point of contact. The impact of variations in the cone and probe geometry is illustrated under the assumptions of invariant mass and moment of inertia, here $m_{r}=1500 \mathrm{~kg}, J_{C_{x}}=J_{C_{y}}=500 \mathrm{~kg} \mathrm{~m}^{2}$ and $J_{T_{x}}=$ $J_{T_{y}}=500 \mathrm{~kg} \mathrm{~m}^{2}$.

Figures (7)-(9) show the stability regions in the dampingdelay and stiffness-delay planes for various values of the length probe, $l_{0}$, the nozzle semi-cone angle, $\theta$, and the distance from the nozzle tip to the contact point, $m^{*}$. Any point lying in the region to the left of a given plot line describes a stable system. The stability region results are helpful in the analysis and design of the stiffness and 

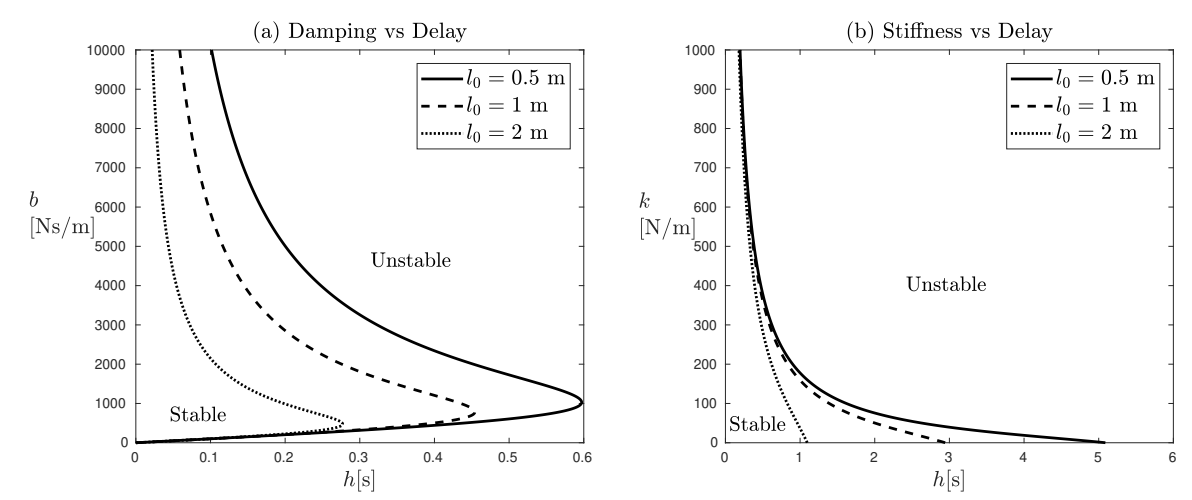

Figure 7. Stability region of stiffness and damping with respect to time-delay for varying probe length ((a) $k=1000 \mathrm{~N} / \mathrm{m}$, (b) $b=200 \mathrm{Ns} / \mathrm{m}, \theta=30^{\circ}$ and $\left.m^{*}=0.5 \mathrm{~m}\right)$
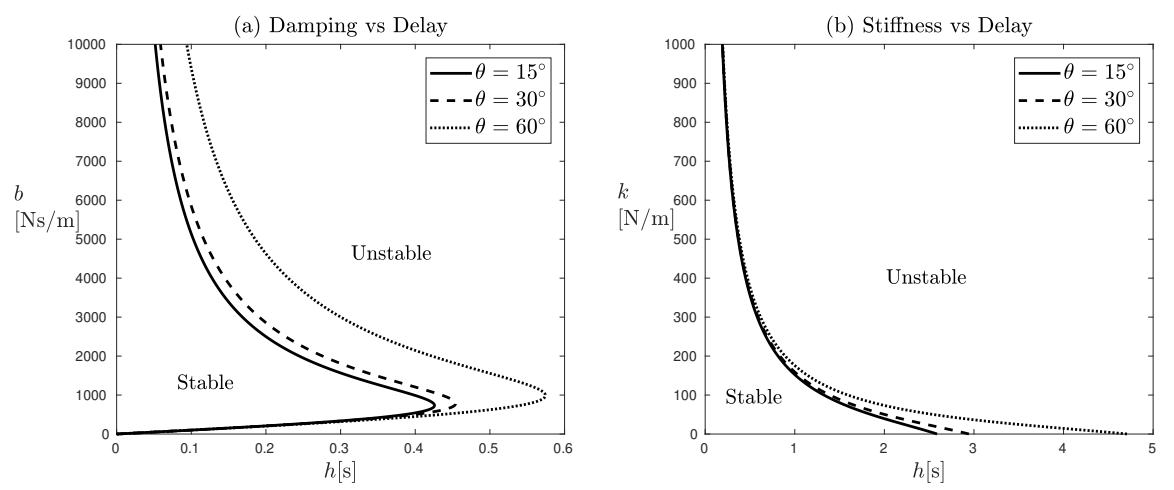

Figure 8. Stability region of stiffness and damping with respect to time-delay for varying semi-cone angle $((\mathrm{a}) k=1000 \mathrm{~N} / \mathrm{m}$, (b) $b=200 \mathrm{Ns} / \mathrm{m}, l_{0}=1 \mathrm{~m}$ and $\left.m^{*}=0.5 \mathrm{~m}\right)$
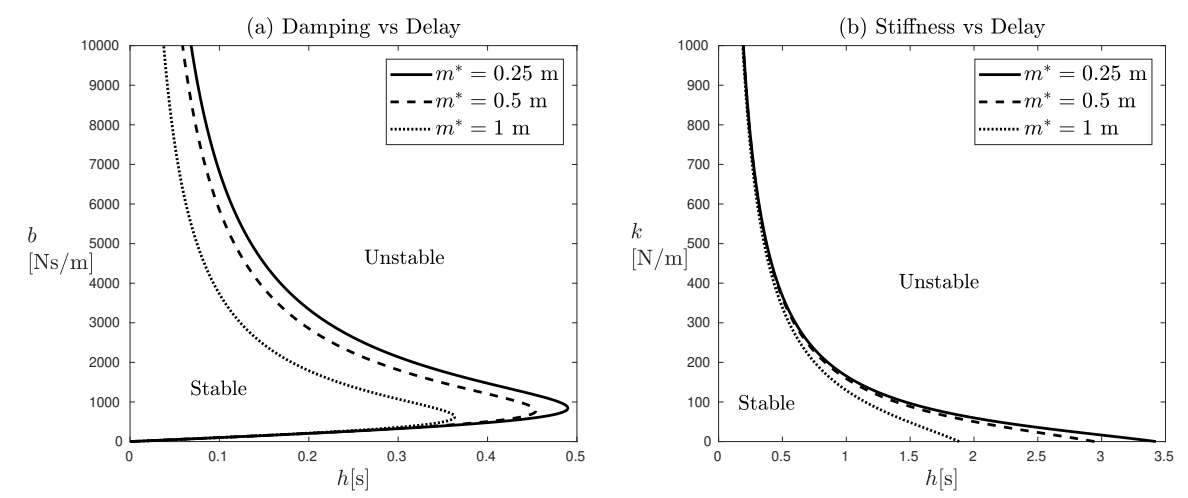

Figure 9. Stability region of stiffness and damping with respect to time-delay for varying distance of contact point from nozzle tip ((a) $k=1000 \mathrm{~N} / \mathrm{m}$, (b) $b=200 \mathrm{Ns} / \mathrm{m}, l_{0}=1 \mathrm{~m}$ and $\theta=30^{\circ}$ )

damping values of the nozzle/probe compliance system that guarantee stability. It is evident from the figures that beyond a certain time delay, the system is unstable for a given cone and probe geometry, irrespective of the compliance parameters. For a relatively smaller value of the time delay, the system is stable for a range of values of the compliance parameters, as illustrated in the plots. A shorter probe length, shorter contact point distance and larger cone angle broadens the range of values of compliance parameters for stability. If the cone and probe geometry are altered, the stability region shifts accordingly, hence can be used to accommodate the unstable set of compliance parameters into the stability region.

\section{Numerical Validation}

\section{Verification}

The nonlinear and linear contact force models provided in Eqs. (59) and (68) respectively are simulated for nonlinear Chaser-Target dynamics in a typical RvD scenario, as provided in Table (1). A constant time delay of $0.016 \mathrm{~s}$ and a nozzle semi-cone angle of $\theta=30^{\circ}$ are assumed for the purpose of simulation. The initial conditions for the simulation are assumed such that the Chaser and Target have $z$-axes in the same direction and $x$-axes in the opposite direction as given in Table (3). The Chaser is moving with 
a relative velocity in $x$ and $y$ directions relative to the Target at $t=0$.

\begin{tabular}{llll}
\hline & Inertial Parameters & \multicolumn{2}{c}{ Geometric Parameters } \\
\hline$m_{r}$ & $1500 \mathrm{~kg}$ & $\mathbf{r}_{T S}$ & $\{1,0,0\}^{T} \mathrm{~m}$ \\
$\mathbf{J}_{C}$ & $\operatorname{diag}(500,500,500) \mathrm{kgm}^{2}$ & $\mathbf{r}_{C Q}$ & $\{2,0,0\}^{T} \mathrm{~m}$ \\
$\mathbf{J}_{T}$ & $\operatorname{diag}(500,500,500) \mathrm{kgm}^{2}$ & $\theta$ & $30^{\circ}$ \\
\hline
\end{tabular}

Table 1. Inertial and geometric parameters for Chaser and Target

\begin{tabular}{ll}
\hline \multicolumn{2}{c}{ Compliance device model parameters } \\
\hline$k_{i}$ & $1000 \mathrm{~N} / \mathrm{m}$ (each) \\
$\mu$ & 0.1 \\
$l_{0}$ & $1 \mathrm{~m}$ \\
$N$ & 4 \\
$a$ & 0.8 \\
$\mathbf{r}_{R S_{1}}$ & $\{0,0,0\}^{T} \mathrm{~m}$ \\
$\mathbf{r}_{R S_{2}}$ & $\{0.2,0,0.2\}^{T} \mathrm{~m}$ \\
$\mathbf{r}_{R S_{3}}$ & $\left\{0.2,0.2 \cos 30^{\circ},-0.2 \sin 30^{\circ}\right\}^{T} \mathrm{~m}$ \\
$\mathbf{r}_{R S_{4}}$ & $\left\{0.2,-0.2 \cos 30^{\circ},-0.2 \sin 30^{\circ}\right\}^{T} \mathrm{~m}$ \\
\hline \multicolumn{2}{c}{ Spring dashpot model parameters } \\
\hline$k$ & $1000 \mathrm{~N} / \mathrm{m}$ \\
$b$ & $200 \mathrm{Ns} / \mathrm{m}$ \\
$\mu$ & 0 \\
\hline
\end{tabular}

Table 2. Geometric and stiffness properties of compliance device model and spring dashpot model

A particular compliance device model is assumed such that it consists of four springs $(N=4)$ of identical stiffness as provided in Table (2). The springs are attached to the probe at point $P$ and to the Chaser at points $R, S_{2}, S_{3}$ and $S_{4}$ as given in the table. The points $S_{2}, S_{3}$ and $S_{4}$ form an equilateral triangle considering a symmetry in the compliance device geometry. For comparison a springdashpot model is assumed with parameters provided in Table (2).

\begin{tabular}{ll}
\hline $\mathbf{r}(0)$ & $\{3.533,0,0.25\}^{T} \mathrm{~m}$ \\
$\mathbf{v}(0)$ & $\{-0.02,0.005,0\}^{T} \mathrm{~m} / \mathrm{s}$ \\
$\mathbf{D}_{\mathcal{C}}^{\mathcal{G}}(0)$ & {$\left[\begin{array}{ccc}-1 & 0 & 0 \\
0 & -1 & 0 \\
0 & 0 & 1\end{array}\right]$} \\
& {$\left[\begin{array}{ccc}1 & 0 & 0 \\
0 & 1 & 0 \\
0 & 0 & 1\end{array}\right]$} \\
$\mathbf{D}_{\mathcal{T}}^{\mathcal{G}}(0)$ & $\{0,0,0\}^{T} \mathrm{rad} / \mathrm{s}$ \\
$\boldsymbol{\omega}_{C}(0)$ & $\{0,0,0\}^{T} \mathrm{rad} / \mathrm{s}$ \\
$\boldsymbol{\omega}_{T}(0)$ & \{
\end{tabular}

Table 3. Initial conditions for simulation

The three dimensional plots of the probe trajectory inside the nozzle are shown in Fig. (10). Each plot correspond to a contact force dynamics model. The probe tip undergoes contact with the nozzle surface at multiple points, the

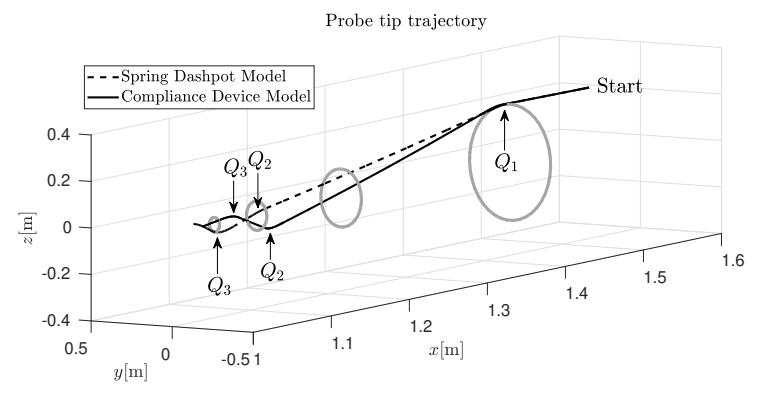

Figure 10. Trajectory of probe tip for two different contact force models

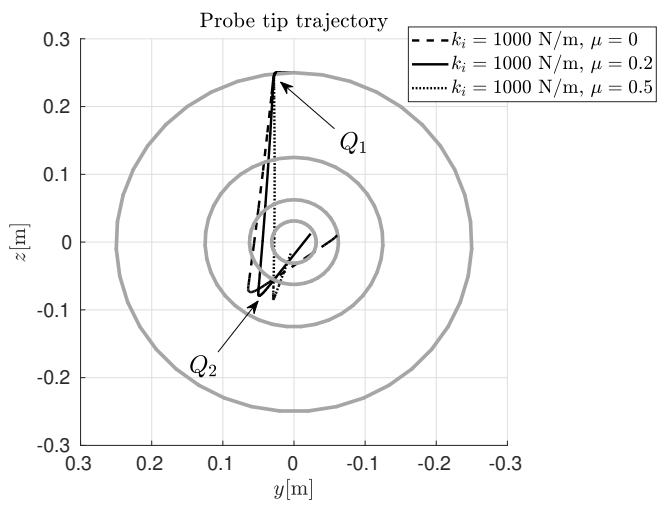

Figure 11. Trajectory of probe tip for compliance device model with a variation in the coefficient of friction

first three of which are marked with $Q_{1}, Q_{2}$ and $Q_{3}$ in chronological order. It can be observed from Fig. (10) that the probe tip in the case of a compliance device model undergoes more angular deviations in the trajectory as compared to other models.

Figures (11) and (12) show the trajectory of the probe tip in the $y z$ and $x z$ plane of the Target frame, respectively, as the coefficient of friction and compliance stiffness varies, for the compliance device model. The shift in the contact point $Q_{2}$ is obvious due to the increase in the friction coefficient. A higher value of coefficient of coefficient of friction, $\mu$, causes an increase in the reaction force and thus in the radial force, which is observed in Fig. (11). Similarly, the shift in the contact point $Q_{2}$ in the $x$ direction of the Target frame in Fig. (12) is due to an increase in the compliance stiffness. The effect of variation in compliance device parameters for the Truth model is therefore verified by the numerical simulations.

\section{Validation}

The stability analysis of the linearized system model in the previous section provides a set of values of the parameters responsible for stability. The stability region results of the linearized model will be validated for the nonlinear model with spring-dashpot contact model. As it is known that the instability associated with time delays in the closed 


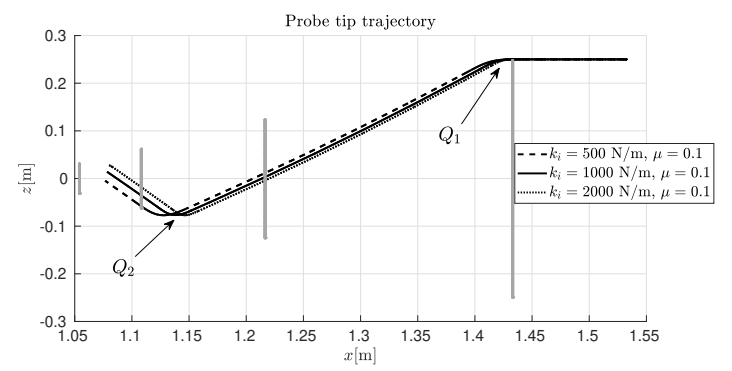

Figure 12. Trajectory of probe tip for the compliance device model with a variation in stiffness

loop is caused by an increase in energy of the system ${ }^{22}$, the kinetic energy of the system is employed to quantify the stability. The use of kinetic energy is justified as it is related with the coefficient of restitution. As opposed to the potential energy of the spring-dashpot, which depends mainly on the modeling assumptions, the kinetic energy is directly computed from the state of the system and the force measurements.

For the case of the spring dashpot contact force model, the kinetic energy follows from the Chaser and Target dynamics given in Eqs. (5)-(8) as

$$
\begin{aligned}
E(t) & =\int_{0}^{t}\left(\mathbf{v}_{C}^{T} \mathbf{f}-\mathbf{v}_{T}^{T} \mathbf{f}\right. \\
& \left.+\boldsymbol{\omega}_{C}^{T} \mathbf{r}_{C Q}^{\times} \mathbf{D}_{\mathcal{C}}^{\mathcal{G}} \mathbf{f}-\boldsymbol{\omega}_{T}^{T} \mathbf{r}_{T Q}^{\times} \mathbf{D}_{\mathcal{T}}^{\mathcal{G}} \mathbf{f}\right) \mathrm{d} t
\end{aligned}
$$

Note that the kinetic energy expression in Eq. (152) represents the increase of kinetic energy with respect to the initial motion at $t=0$. Based on the kinetic energy, the system is passive, lossless and active when $E<0, E=0$ and $E>0$, respectively, after the impact ${ }^{30}$.

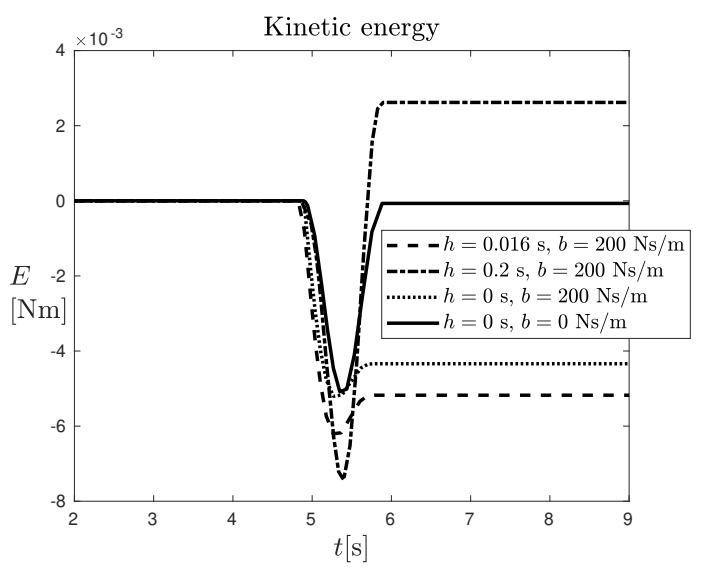

Figure 13. Kinetic energy for the two cases in comparison with the zero-delay case

There are two test cases considered emerging from the stability region shown in Figs. (7)-(9). Stiffness and damping parameters of $1000 \mathrm{~N} / \mathrm{m}$ and $200 \mathrm{Ns} / \mathrm{m}$, respectively, yield a stable system when the delay is $0.016 \mathrm{~s}$, but an unstable one when it is $0.2 \mathrm{~s}$. In both the cases the other parameters, namely $\theta=30^{\circ}, l_{0}=1 \mathrm{~m}$ and $m^{*}=0.5 \mathrm{~m}$, are kept identical. The kinetic energy for the nonlinear system during the first contact is shown in Fig. (13) for the two cases.

The solid line and the dotted line in Fig. (13) show the kinetic energy for an ideal simulation case of elastic impact $(b=0 \mathrm{Ns} / \mathrm{m})$ and a damped impact $(b=200 \mathrm{Ns} / \mathrm{m})$, respectively. The dip in the curves during the contact is due to the transfer of the kinetic energy to the potential energy in the springs and the dissipated energy via the dampers of the system. It can be observed that, for the ideal simulation of the damped impact in dotted line, the system experienced energy dissipation due to damping. The case of damped impact with a $0.016 \mathrm{~s}$ delay in dashed line shows a negative energy after the impact, therefore the nonlinear system is stable. In the case of a $0.2 \mathrm{~s}$ delay, shown in dot-dashed line, there is an added energy to the system, leading to a positive energy after the impact, therefore the nonlinear system is unstable. This validates the linearized stability analysis of the nonlinear model.

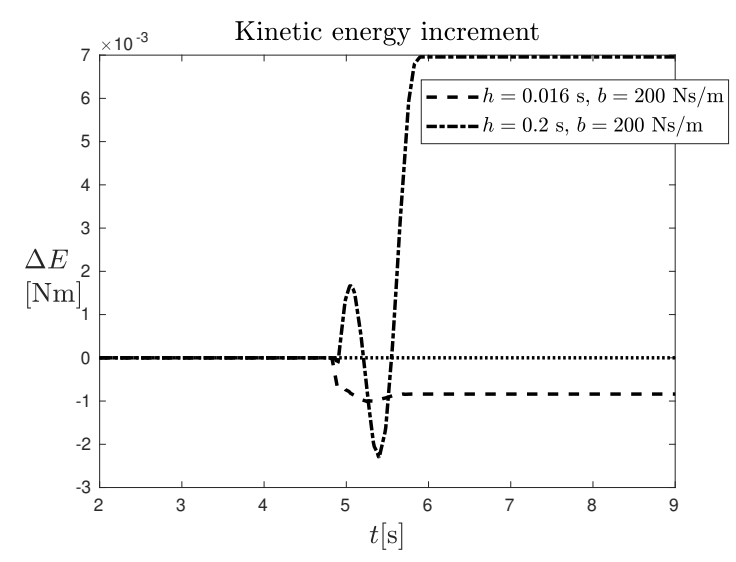

Figure 14. Kinetic energy increment for the two cases with respect to the zero-delay case

A comparison of the two cases with respect to the ideal simulation of damped impact ( $h=0 \mathrm{~s}, b=200 \mathrm{Ns} / \mathrm{m}$ ) is shown in Fig. (14). It can be observed that for the case of a $0.2 \mathrm{~s}$ delay, the system has gained an additional $7 \times 10^{-3}$ $\mathrm{Nm}$ energy with respect to the ideal case due to the time delay in the loop. A time delay of $0.016 \mathrm{~s}$ led to an energy dissipation of $8 \times 10^{-4}$ with respect to the ideal simulation, which although guarantees the stability of contact, but does not reflect the contact dynamics of ideal simulation.

\section{Conclusion}

The present work has addressed the problem of instability in contact during Rendezvous and Docking simulations in the presence of a time delay in the system. The modeling 
of a two body system, namely, Chaser and Target is carried out in three dimensions. A nonlinear contact force model is presented based on a generic compliance device mounted on the Chaser body, and the resulting contact force is shown to be a function of the system states. A surface friction force at the contact point in accordance with the Coulomb friction model is also considered. The trajectory of the contact point is simulated and compared with the traditional springdashpot contact force model. The effects of variation in the surface friction and stiffness are also numerically studied.

The Chaser and Target system is linearized considering a spring-dashpot model and a reduced set of states, namely penetration depth, azimuthal angle and distance of the contact point from the tip on the nozzle surface are derived. Further, the stability region in terms of the stiffness, damping, and delay parameters is derived for a varying probe and cone geometry. The numerical validation via energy considerations shows that the linearized analysis gives a satisfying prediction of the nonlinear system stability.

\section{Conflict of Interest}

The Authors declare that there is no conflict of interest.

\section{Funding}

This research received no specific grant from any funding agency in the public, commercial, or not-for-profit sectors.

\section{References}

1. Polites ME. An Assessment of the Technology of Automated Rendezvous and Capture in Space. 1998.

2. Fehse W. Automated Rendezvous and Docking of Spacecraft. 1st Edition: Cambridge University Press, 2003.

3. Zimpfer D, Kachmar P and Tuohy S. Autonomous Rendezvous, Capture and In-Space Assembly:Past, Present and Future. In: 1st Space Exploration Conference: Continuing the Voyage of Discovery, 30 Jan 2005, pp. 2523.

4. Flores-Abad A, Ma O, Pham K and Ulrich S. A review of space robotics technologies for on-orbit servicing. Progress in Aerospace Sciences. 2014; 68:1-26.

5. Yoshida K. Ets-vii flight experiments for space robot dynamics and control. In: Experimental Robotics VII, 2001, pp. 209-218. Springer, Berlin, Heidelberg.

6. Friend RB. Orbital Express program summary and mission overview. In: Sensors and Systems for space applications II, 2008, Vol. 6958, pp. 695803. International Society for Optics and Photonics.
7. Coleshill E, Oshinowo L, Rembala R, Bina B, Rey D and Sindelar S. Dextre: Improving maintenance operations on the international space station. Acta Astronautica. 2009; 64(910):869-874.

8. Stoll E, Walter U, Artigas J, Preusche C, Kremer P, Hirzinger G, Letschnik J and Pongrac H. Ground verification of the feasibility of telepresent on-orbit servicing. Journal of Field Robotics. 2009; 26(3):287-307.

9. Schwartz JL, Peck MA and Hall CD. Historical review of spacecraft simulators. Journal of Guidance, Control, and Dynamics. 2003; 26(4):513-522

10. Zebenay M, Boge T, Krenn R and Choukroun D. Analytical and experimental stability investigation of a hardware-in-theloop satellite docking simulator. Proceedings of the Institution of Mechanical Engineers, Part G: Journal of Aerospace Engineering. 2015; 229(4):666-681.

11. Chapleau SL, Martin E and Baron L. Results and Verification of Spacecraft Docking Emulation using Hardware-in-the-Loop Simulation. In: Romansy 16: Robot Design, Dynamics, and Control, 2006, pp. 397-404. Springer Vienna

12. Paul J, Dettmann A, Girault B, Hilljegerdes J, Kirchner F, Ahrns I and Sommer J. INVERITAS: A facility for hardwarein-the-loop long distance movement simulation for rendezvous and capture of satellites and other autonomous objects. Acta Astronautica. 2015; 116:1-24

13. Rybus $T$ and Seweryn K. Planar air-bearing microgravity simulators: Review of applications, existing solutions and design parameters. Acta Astronautica. 2016; 120:239-259.

14. Regoli L, Herrmann C, Ravandoor K and Schilling K. New Testing Facility for Proximity Operations in Space. IFAC Proceedings Volumes. 2013; 46(19):353-358.

15. Ma O, Flores-Abad A and Boge T. Use of industrial robots for hardware-in-the-loop simulation of satellite rendezvous and docking. Acta Astronautica. 2012; 81(1):335-347.

16. Mandal $\mathrm{N}$ and Payandeh S. Experimental evaluation of the importance of compliance for robotic impact control. In: Control Applications, Second IEEE Conference on, Vancouver, BC, 13 Sep 1993, pp. 511-516. IEEE.

17. Gilardi $G$ and Sharf I. Literature survey of contact dynamics modelling. Mechanism and Machine Theory. 2002; 37(10):1213-1239.

18. Ma O. Contact dynamics modelling for the simulation of the Space Station manipulators handling payloads. In: Robotics and Automation, Proceedings, IEEE International Conference on, Nagoya, 1995, Vol. 2, pp. 1252-1258.

19. Ma O and Wang J. Model order reduction for impact-contact dynamics simulations of flexible manipulators. Robotica. 2007; 25(4):397-407. 
20. Bondoky K, Janschek K, Rathke A and Schwarz S. Analysis of Hardware-in-the-Loop setup without artificial compliance for docking contact dynamics of satellites. In: AIAA SPACE and Astronautics Forum and Exposition, 2017, pp. 5183

21. Chang T, Cong D, Ye Z and Han J. Time Problems in HIL Simulation for On-orbit Docking and Compensation. In: Industrial Electronics and Applications, 2nd IEEE Conference on, Harbin, 2007, pp. 841-846. IEEE.

22. Osaki K, Konno A and Uchiyama M. Delay time compensation for a hybrid simulator. Advanced Robotics. 2010; 24(89):1081-1098.

23. Abiko S, Satake Y, Jiang X, Tsujita T and Uchiyama M. Delay time compensation based on coefficient of restitution for collision hybrid motion simulator. Advanced Robotics. 2014; 28(17):1177-1188.

24. Qi C, Zhao X, Gao F, Ren A and Sun Q. Contact stiffness and damping identification for hardware-in-the-loop contact simulator with measurement delay compensation. Acta Astronautica. 2016; 123:171-180.

25. Qi C, Gao F, Zhao X, Ren A and Wang Q. A force compensation approach toward divergence of Hardwarein-the-Loop contact simulation system for damped elastic contact. IEEE Transactions on Industrial Electronics. 2017; 64(4):2933-2943.

26. Wahbah M. On the dynamics and control of space contact simulators. In: Guidance, Navigation, and Control Conference, American Institute of Aeronautics and Astronautics, Baltimore, MD, U.S.A., 1995, pp. 3339.

27. Glocker $\mathrm{C}$ and Pfeiffer F. Complementarity problems in multibody systems with planar friction. Archive of Applied Mechanics, Springer-Verlag. 1993; 63(7):452-463.

28. Zebenay M, Boge T, Choukroun D. Modeling and stability analysis of a hybrid docking simulator. In: Guidance, Navigation, and Control Conference, American Institute of Aeronautics and Astronautics, Boston, MA, U.S.A., 2013.

29. Marshall JE, Gorecki H, Korytowski A and Walton K. Time-delay systems: Stability and performance criteria with applications. 1st ed. London: Ellis Horwood, 1992

30. Khalil HK. Nonlinear systems. Prentice-Hall, New Jersey, 1996

\section{Appendix-A}

The penetration rate can be written from Eq. (42) as

$$
\dot{d}=\mathbf{v}^{T} \mathbf{D}_{\mathcal{G}}^{\mathcal{N}} \hat{\mathbf{n}}+\mathbf{r}^{T} \dot{\mathbf{D}}_{\mathcal{G}}^{\mathcal{N}} \hat{\mathbf{n}}+\mathbf{r}_{C Q}^{T} \dot{\mathbf{D}}_{\mathcal{C}}^{\mathcal{N}} \hat{\mathbf{n}}-\mathbf{r}_{T S}^{T} \dot{\mathbf{D}}_{\mathcal{T}}^{\mathcal{N}} \hat{\mathbf{n}}
$$

In order to find the penetration rate, the time derivative of Nozzle frame attitude with respect to inertial, Chaser and Target frames, $\dot{\mathbf{D}}_{\mathcal{G}}^{\mathcal{N}}, \dot{\mathbf{D}}_{\mathcal{C}}^{\mathcal{N}}$ and $\dot{\mathbf{D}}_{\mathcal{T}}^{\mathcal{N}}$, respectively. In the present model, the Nozzle frame is known to be rotating with angular velocity $\dot{\phi}$ with respect to the Target frame about $x_{\mathcal{T}}$-axis

$$
\dot{\mathbf{D}}_{\mathcal{T}}^{\mathcal{N}}=\boldsymbol{\omega}_{N}^{\times} \mathbf{D}_{\mathcal{T}}^{\mathcal{N}}
$$

where, $\boldsymbol{\omega}_{N}=\{\dot{\phi}, 0,0\}^{T}$. The time derivative of azimuthal angle can be found from Eq. (30) as

$$
\dot{\phi}=\frac{\left[\hat{\mathbf{y}}_{\mathcal{T}}^{T}+\hat{\mathbf{z}}_{\mathcal{T}}^{T} \tan \phi\right] \dot{\mathbf{r}}_{S Q}}{\left[\hat{\mathbf{y}}_{\mathcal{T}}^{T} \tan \phi-\hat{\mathbf{z}}_{\mathcal{T}}^{T}\right] \mathbf{r}_{S Q}}
$$

The time derivative of Nozzle frame attitude with respect to Chaser and Target frames can be written using Eqs. (52) and (65) as

$$
\begin{aligned}
\dot{\mathbf{D}}_{\mathcal{G}}^{\mathcal{N}} & =\dot{\mathbf{D}}_{\mathcal{G}}^{\mathcal{T}} \mathbf{D}_{\mathcal{T}}^{\mathcal{N}}+\mathbf{D}_{\mathcal{G}}^{\mathcal{T}} \dot{\mathbf{D}}_{\mathcal{T}}^{\mathcal{N}} \\
& =\mathbf{D}_{\mathcal{G}}^{\mathcal{T}}\left[\boldsymbol{\omega}_{T}+\boldsymbol{\omega}_{N}\right]^{\times} \mathbf{D}_{\mathcal{T}}^{\mathcal{N}} \\
\dot{\mathbf{D}}_{\mathcal{C}}^{\mathcal{N}} & =\dot{\mathbf{D}}_{\mathcal{C}}^{\mathcal{G}} \mathbf{D}_{\mathcal{G}}^{\mathcal{N}}+\mathbf{D}_{\mathcal{C}}^{\mathcal{G}} \dot{\mathbf{D}}_{\mathcal{G}}^{\mathcal{N}} \\
& =-\boldsymbol{\omega}_{C}^{\times} \mathbf{D}_{\mathcal{C}}^{\mathcal{N}}+\mathbf{D}_{\mathcal{C}}^{\mathcal{G}} \mathbf{D}_{\mathcal{G}}^{\mathcal{T}}\left[\boldsymbol{\omega}_{T}+\boldsymbol{\omega}_{N}\right]^{\times} \mathbf{D}_{\mathcal{T}}^{\mathcal{N}}
\end{aligned}
$$

\section{Appendix-B}

The dynamics of the Chaser-Target system can be expressed in terms of $\delta \mathbf{r}_{S Q}$ by substituting $\delta d$ and $\delta \dot{d}$ from Eqs. (113) and (114) into Eq. (112), and $f_{n}$ from Eq. (112) into Eqs. (109)-(111) as follows

$$
\begin{aligned}
& \boldsymbol{\delta} \dot{\mathbf{v}}=-\frac{\hat{\mathbf{n}}_{\mathcal{T}}^{*} \hat{\mathbf{n}}_{\mathcal{T}}^{* T}}{m_{r}}\left[b \boldsymbol{\delta} \dot{\mathbf{r}}_{S Q}(t-h)\right. \\
& \left.+k \delta \mathbf{r}_{S Q}(t-h)\right] \\
& \boldsymbol{\delta} \dot{\boldsymbol{\omega}}_{C}=-\mathbf{J}_{C}^{-1} \mathbf{P}_{C}^{T} \hat{\mathbf{n}}_{\mathcal{T}}^{*} \hat{\mathbf{n}}_{\mathcal{T}}^{* T}\left[b \delta \dot{\mathbf{r}}_{S Q}(t-h)\right. \\
& \left.+k \boldsymbol{\delta} \mathbf{r}_{S Q}(t-h)\right] \\
& \boldsymbol{\delta} \dot{\boldsymbol{\omega}}_{T}=-\mathbf{J}_{T}^{-1}\left(\mathbf{P}_{T}^{T}+\mathbf{r}_{T S}^{\times}\right) \hat{\mathbf{n}}_{\mathcal{T}}^{*} \hat{\mathbf{n}}_{\mathcal{T}}^{* T}\left[b \boldsymbol{\delta} \dot{\mathbf{r}}_{S Q}(t-h)\right. \\
& \left.+k \delta \mathbf{r}_{S Q}(t-h)\right]
\end{aligned}
$$

Using Eq. (116), the dynamics of $\delta \mathbf{r}_{S Q}$ can be written as

$$
\delta \ddot{\mathbf{r}}_{S Q}=\delta \dot{\mathbf{v}}+\mathbf{P}_{C} \delta \dot{\boldsymbol{\omega}}_{C}+\mathbf{P}_{T} \boldsymbol{\delta} \dot{\boldsymbol{\omega}}_{T}
$$

Substituting the dynamic equations of the Chaser-Target from Eqs. (158)-(160) gives

$$
\delta \ddot{\mathbf{r}}_{S Q}(t)=-\mathbf{B} \boldsymbol{\delta} \dot{\mathbf{r}}_{S Q}(t-h)-\mathbf{K} \boldsymbol{\delta} \mathbf{r}_{S Q}(t-h)
$$

for some constant matrices $\mathbf{B}$ and $\mathbf{K}$. It can be noticed that spacecraft system dynamics in Eqs. (158)-(160) is a linear combination of the states of system described by Eq. (162). Therefore, the stability of the system in Eq. (162) implies the stability of the spacecraft system. 